\title{
Flight Control of a Hexa-Rotor Airship: Uncertainty Quantification for a Range of Temperature and Pressure Conditions
}

\author{
Davi Antonio dos Santos ${ }^{\mathrm{a}}$, Americo Cunha $\mathrm{Jr}^{\mathrm{b}}$ \\ ${ }^{a}$ Aeronautics Institute of Technology, Sao Jose dos Campos, Brazil \\ ${ }^{b}$ Rio de Janeiro State University, Rio de Janeiro, Brazil
}

\begin{abstract}
The present paper is concerned with the dynamic modeling and design of control laws for a small non-rigid multi-rotor airship constituted of an oblate-spheroid helium balloon coupled with an electric-powered hexa-rotor airframe. The vehicle is assumed to operate in windless and lowspeed conditions. A six-degree-of-freedom nonlinear dynamic model is derived for it using the Newton-Euler approach and considering, among other common efforts, a restoring torque due to the displacement of the balloon's center of buoyancy above the vehicle's center of mass and the added-mass effect resulting from the air-structure interaction. Using the derived model and assuming a time-scale separation between the translational and rotational dynamics, the attitude and position control laws are designed separately from each other. Both laws are formulated using feedback linearization combined with control input saturation within appropriate parallelepipedal sets, which are carefully chosen to respect pre-defined bounds on the control torque, control force and inclination angle. The effect of temperature and pressure fluctuations is taken into account through a parametric probabilistic approach, where Maximum Entropy Principle is used to construct a physically consistent stochastic model and Monte Carlo method is used as stochastic solver to propagate the uncertainties through the system. Extensive simulation results show the effectiveness of the proposed control system and quantifies the uncertainty of its performance over a wide range of local temperature and pressure.

Keywords: multi-rotor airship, multi-rotor aerial vehicle, flight control system, uncertainty quantification.
\end{abstract}




\section{Introduction}

The traditional airships are lighter-than-air (LTA) aircrafts constituted of a streamlined or elliptical envelop (or hull) filled with a low-density gas (generally, helium), a pair of propellers installed through the gondola on its bottom part and a tail equipped with rudder and elevator. They mainly rely on the aerostatic lift, resulting from the difference between the air and inner gas densities, for opposing the gravity and keep flying, which provides them with better endurance, payload capacity, and energy consumption characteristics compared to heavier-than-air (HTA) vehicles (such as airplanes and helicopters) [1].

In the last three decades, thanks to advances (and cost reduction) in sensor technology, embedded computers, and materials, the airships have resurged in academia, mainly in non-rigid (blimp), small, and unmanned versions [2]. Such interest renewal in turn gives rise to many works concerned with the dynamic modeling and identification $[3,4]$ as well as the flight control $[5,6]$ of such aerial vehicles.

Despite of the aforementioned advantages of the traditional airships over the HTA vehicles, they suffer from a weak controllability in the vertical and lateral translational degrees of freedom (DOF), besides of being more vulnerable to undesirable aerodynamic effects, such as those due to the drag and the added mass [1]. The interplay between these drawbacks has motivated the design of a number of small and electric hybrid airships $[7,8,9,10]$ provided with an augmented maneuverability by an unconventional combination of actuators. In particular, the reference [7] presents a fin-less ellipsoidal blimp equipped with four vectoring rotors; the reference [8] analyzes a small streamlined blimp actuated by one vertical and two horizontal fixed rotors; the reference [9] is concerned with a flying-wing-shaped hull equipped with four vectoring double rotors; and the reference [10] comes up with a cylindrical blimp with conventional actuators, whose rotor axis can be controlled to slide along a longitudinal keel.

The present paper investigates the dynamic modeling and flight control of a hexa-rotor airship, which is a new hybrid aircraft consisting of an oblate-spheroid balloon filled with helium, attached to a hexa-rotor airframe (see a picture in Figure 1). Their two parts are strapped down by ropes at six contact points. The balloon is made on polyurethane and has $2.5 \mathrm{~m}$ of horizontal diameter and 
$1.6 \mathrm{~m}$ of height. With a payload of $4.42 \mathrm{~kg}$, the vehicle has a buoyancy ratio of 0.7 . In a qualitative pre-analysis, one can say that, in low speed and windless condition, the hexa-rotor airship has potentially a better flight duration and payload capacity than a pure hexa-rotor vehicle, since its aerostatic lift is sized to counteract part of the weight aboard it. On the other hand, compared to other airships, the one investigated here has a full maneuverability in the position and heading DOFs, which is a characteristic inherited from conventional multi-rotor vehicles.

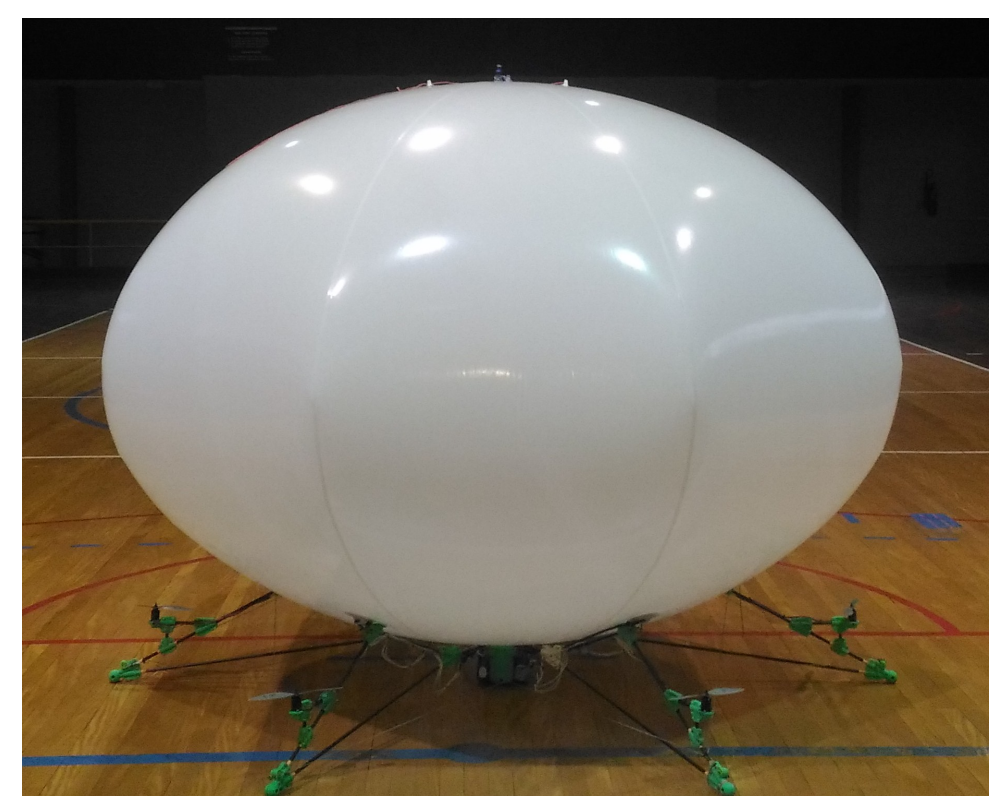

Figure 1: The hexa-rotor airship. It is patented according to reference [11].

In particular, the present paper is concerned with the dynamic modeling and design of flight control laws for the hexa-rotor airship depicted in Figure 1. A nonlinear six-DOF dynamic model is derived for this vehicle using the Newton-Euler approach, considering its operation in a lowspeed and windless condition. On the basis of this model and considering a time-scale separation between the translational and rotational dynamics, a hierarchical flight control architecture with the attitude control loop put inside the position control loop is adopted here. This architecture is commonly found in the literature of control applied to multi-rotor aerial vehicles (MAV) $[12,13,14]$. Here, on the basis of the derived model, the attitude and position control laws are separately designed using feedback linearization and considering the saturation of the control vector within appropriate parallelepipedal sets that ensure the satisfaction of design bounds on the control torque 
and force, as well as on the vehicle's inclination angle.

The design of position and attitude control laws for the hexa-rotor airship, even if it is intended to operate indoors and in low speed, must face two paramount dynamic aspects not found in the MAV control problem. The first one is the restoring torque, which appears as a consequence of the displacement between the balloon's center of buoyancy (CB) and the vehicle's center of mass (CM) [15]. For a fixed CB-CM displacement, the larger the inclination angle of the vehicle with respect to the local vertical, the larger the magnitude of the restoring torque. Therefore, for a given sizing of the rotor set, the attitude controller must respect a maximum bound on the inclination angle for the control system to maintain its effectiveness to counteract the restoring torque (and more). The side effect of such inclination constraint is a low lateral acceleration capability. The second aspect is the added-mass effect [16], which consists in the reaction efforts (torque and force) of the surrounding air on the vehicle's body. It turns out that the added-mass effect causes an additional coupling between the translational and rotational dynamics, besides an apparent increasing in the vehicle's mass and inertia. The above two aspects are considered here. Moreover, an uncertainty quantification analysis is carried out to assess the variation in the closed-loop performance as a result of the deviation of the operating temperature and pressure with respect to their nominal values; note that such deviation causes a change in the air and helium densities and, as a consequence, in the aerostatic lift and restoring torque. From the above justification, we can assert that the main contributions of this paper are: 1) the dynamic modeling of the hexa-rotor airship for windless and low-speed conditions; 2) the design of a simple but effective control scheme for this vehicle, considering the aerostatic efforts; and 3) the uncertainty quantification study for assessing its closed-loop performance over a specified range of possible operating temperatures and pressures.

The remaining text is organized in the following manner. Section 2 derives the six-DOF dynamic model. Section 3 is concerned with the design of the nonlinear attitude and position control laws, as well as the stability analysis of the corresponding closed-loop dynamics. Sections 4 and 5 evaluates the overall system numerically. Particularly, Section 4 adopts a nominal scenario, whereas Section 5 carries out a stochastic uncertainty quantification analysis considering a random variation of both the local temperature and pressure conditions over given ranges. Finally, Section 6 concludes 

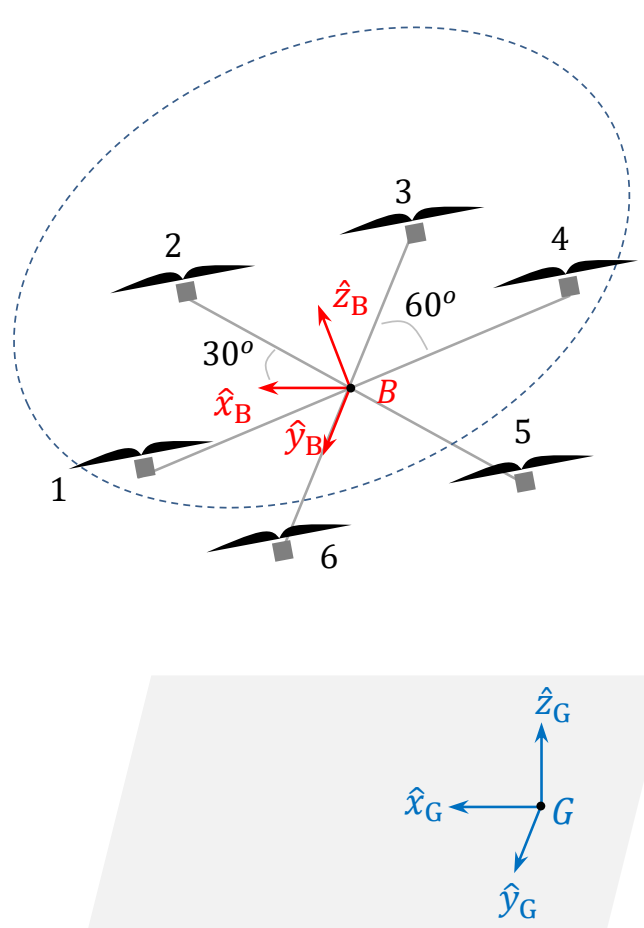

Figure 2: Illustration of the hexa-rotor airship and representaiton of the Cartesian coordinate systems.

the paper.

\section{Dynamic Modeling}

This section derives rotational and translational equations of motion, as well as actuator models for the hexa-rotor airship, considering the most relevant efforts in low-speed indoor flight scenarios. We start with preliminary definitions in Subsection 2.1, then we model the rotor dynamics and control efforts in Subsection 2.2, the restoring torque and aerostatic lift generated by the balloon in Subsection 2.3, the vehicle's translational dynamics in Subsection 2.5, and its rotational dynamics in Subsection 2.4. Finally, the added-mass effects are treated in Subsection 2.6.

\subsection{Preliminary Definitions, Notation, and Nomenclature}

Define two Cartesian coordinate systems (CCS) as illustrated in Figure 2. The body CCS, $\mathcal{S}_{\mathrm{B}} \triangleq\left\{B ; \hat{x}_{\mathrm{B}}, \hat{y}_{\mathrm{B}}, \hat{z}_{\mathrm{B}}\right\}$ is attached to the vehicle's body with the origin at the vehicle's center of mass $B$, the $\hat{x}_{\mathrm{B}}$ axis is pointing forward, aligned with the bisectrix of the separation angle between 
the rotors 1 and 2 , the $\hat{z}_{\mathrm{B}}$ axis is pointing upward, normal to the airframe, and the $\hat{y}_{\mathrm{B}}$ axis completes a right-handed coordinate system. The ground CCS, $\mathcal{S}_{\mathrm{G}} \triangleq\left\{G ; \hat{x}_{\mathrm{G}}, \hat{y}_{\mathrm{G}}, \hat{z}_{\mathrm{G}}\right\}$ is fixed to the ground at a known point $G$, with the $\hat{z}_{\mathrm{G}}$ axis pointing upward vertically. For our purposes, $\mathcal{S}_{\mathrm{G}}$ can be considered as an inertial frame and will be adopted as such.

Denote the set of real numbers by $\mathbb{R}$ and the set of positive and non-negative real numbers by $\mathbb{R}_{>0}$ and $\mathbb{R}_{\geq 0}$, respectively. Moreover, denote the special orthogonal group by $\mathrm{SO}(3)$. Denote arbitrary sets by uppercase calligraphic letters, e.g., $\mathcal{A}$ and $\mathcal{B}$. The Minkowski sum of two sets $\mathcal{A}$ and $\mathcal{B}$ is defined by $\mathcal{A} \oplus \mathcal{B} \triangleq\{a+b: a \in \mathcal{A}, b \in \mathcal{B}\}$. To clarify the vector notation adopted here, consider two different kinds of vectors: physical (geometric) vectors and algebraic vectors. Physical vectors are denoted by italic letters with a right arrow superscript, e.g., $\vec{a}$. The corresponding algebraic vector resulting from the projection of $\vec{a}$ onto an arbitrary CCS $\mathcal{S}_{\mathrm{A}}$ is denoted by the same letter, but in a bold format, with the subscript $A$, i.e., $\mathbf{a}_{A} \in \mathbb{R}^{3}$. Matrices are denoted by bold uppercase letters, e.g., A. Let $\vec{a}$ be an arbitrary physical vector. The relation between its representations $\mathbf{a}_{\mathrm{A}}$ and $\mathbf{a}_{\mathrm{B}}$ is $\mathbf{a}_{\mathrm{A}}=\mathrm{D}^{\mathrm{A} / \mathrm{B}} \mathbf{a}_{\mathrm{B}}$, where $\mathbf{D}^{\mathrm{A} / \mathrm{B}} \in \mathrm{SO}(3)$ is the attitude matrix of $\mathcal{S}_{\mathrm{A}}$ w.r.t. $\mathcal{S}_{\mathrm{B}}$. The inverse of $\mathbf{D}^{\mathrm{A} / \mathrm{B}}$ (which coincides with its transpose) is sometimes denoted by $\mathbf{D}^{\mathrm{B} / \mathrm{A}}$. The Euclidean norm of $\mathbf{a}_{\mathrm{A}}$ is denoted by $\left\|\mathbf{a}_{\mathrm{A}}\right\|$. Now, let $\vec{a}^{\mathrm{A} / \mathrm{B}}$ represent a physical quantity of $\mathcal{S}_{\mathrm{A}}$ relative to $\mathcal{S}_{\mathrm{B}}, e . g$., $\vec{r}^{\mathrm{B} / \mathrm{G}}$ denotes the relative position of $\mathcal{S}_{\mathrm{B}}$ w.r.t. $\mathcal{S}_{\mathrm{G}}$. Let $\mathbf{e}_{i}$ denote an $n$-dimensional (for some $n$ ) standard unit vector. The $i$ th component of $\mathbf{a}$ is denoted by $a_{i}$ and sometimes by $\mathbf{e}_{i}^{\mathrm{T}} \mathbf{a}$. Now, consider two physical vectors $\vec{a}$ and $\vec{b}$ as well as the respective algebraic representations in $\mathcal{S}_{\mathrm{A}}$, $\mathbf{a}_{\mathrm{A}}=\left[\begin{array}{lll}a_{1} & a_{2} & a_{3}\end{array}\right]^{\mathrm{T}}$ and $\mathbf{b}_{\mathrm{A}}$, respectively. Denote the $\mathcal{S}_{\mathrm{A}}$ representation of the vector product $\vec{a} \times \vec{b}$ by the matrix multiplication $\left[\mathbf{a}_{\mathrm{A}} \times\right] \mathbf{b}_{\mathrm{A}}$, where $\left[\mathbf{a}_{\mathrm{A}} \times\right]$ is the following skew-symmetric matrix:

$$
\left[\mathbf{a}_{\mathrm{A}} \times\right] \triangleq\left[\begin{array}{ccc}
0 & -a_{3} & a_{2} \\
a_{3} & 0 & -a_{1} \\
-a_{2} & a_{1} & 0
\end{array}\right]
$$

The text also distinguishes between a variable representing an effective quantity, e.g., $a$, and the corresponding command (or desired value). The latter is denoted with the same letter as the effective quantity, but including an overbar; e.g., $\bar{a}$. 


\subsection{Rotor Dynamics and Efforts}

The set of six rotors equipping the airframe is responsible for generating the control forces and torques as described here. The $i$ th rotor individually produces a thrust force and a reaction torque on the airframe along the $\hat{z}_{\mathrm{B}}$ axis with magnitudes denoted by $f_{i}$ and $\tau_{i}$, respectively. We describe these efforts by the following aerodynamic models [17]:

$$
\begin{gathered}
f_{i}=k_{f} \omega_{i}^{2}, \\
\tau_{i}=k_{\tau} \omega_{i}^{2},
\end{gathered}
$$

$i=1, \ldots, 4$, where $k_{f}$ is the thrust force coefficient, $k_{\tau}$ is the reaction torque coefficient, and $\omega_{i}$ is the rotation speed of the $i$ th rotor. The rotor dynamics can be modeled by the following first-order linear model:

$$
\dot{\omega}_{i}=-\frac{1}{\tau_{\omega}} \omega_{i}+\frac{k_{\omega}}{\tau_{\omega}} \bar{\omega}_{i}
$$

where $\bar{\omega}_{i} \in\left[0, \bar{\omega}_{\max }\right]$ is the rotation speed command of the $i$ th rotor, $k_{\omega}$ is the speed coefficient, and $\tau_{\omega}$ is the rotor time constant. The rotation upper bound $\bar{\omega}_{\max }$ is assumed to be known.

Consider that all the six thrusts $f_{i}$ point upward. Moreover, consider that the reaction torque $\tau_{1}$ is positive, $\tau_{2}$ is negative, $\tau_{3}$ is positive, and so on. Therefore, one can show that the magnitude $F^{c}$ of the resulting control force and the $\mathcal{S}_{\mathrm{B}}$ representation $\mathbf{T}_{\mathrm{B}}^{c}$ of the resulting control torque are given by

$$
\left[\begin{array}{l}
F^{c} \\
\mathbf{T}_{\mathrm{B}}^{c}
\end{array}\right]=\boldsymbol{\Gamma \mathbf { f }}
$$

where $\mathbf{f} \triangleq\left[\begin{array}{llll}f_{1} & f_{2} & \ldots & f_{6}\end{array}\right]^{\mathrm{T}}$ and 

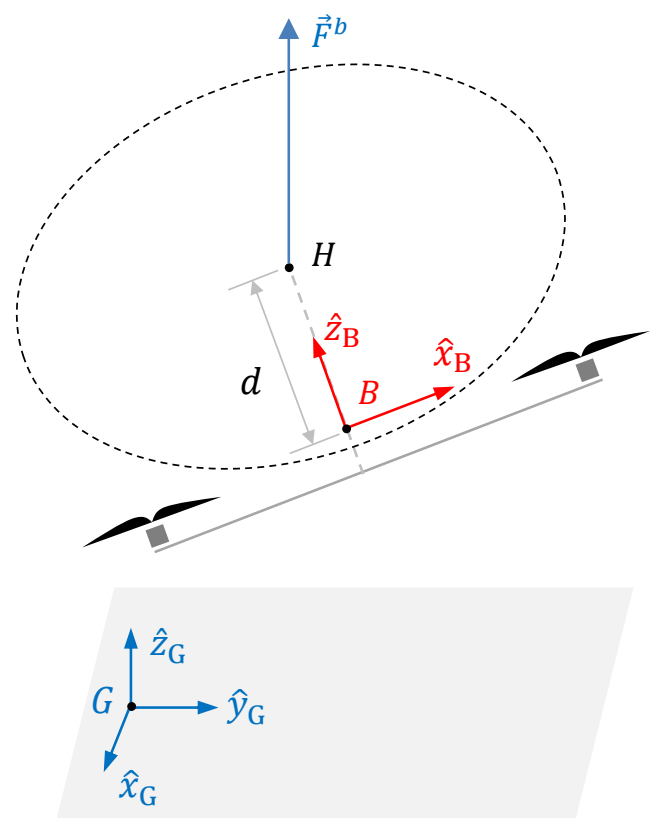

Figure 3: The restoring torque.

$$
\Gamma \triangleq\left[\begin{array}{rrrrrr}
1 & 1 & 1 & 1 & 1 & 1 \\
l / 2 & -l / 2 & -l & -l / 2 & l / 2 & l \\
-l \sqrt{3} / 2 & -l \sqrt{3} / 2 & 0 & l \sqrt{3} / 2 & l \sqrt{3} / 2 & 0 \\
k & -k & k & -k & k & -k
\end{array}\right] \in \mathbb{R}^{4 \times 6}
$$

with $l$ denoting the length of each vehicle's arm, and $k \triangleq k_{\tau} / k_{f}$.

\subsection{Aerostatic Lift and Restoring Torque}

This subsection models two crucial efforts generated by the balloon. One is an aerostatic lift force $\vec{F}^{b}$ and the other one is a restoring torque $\vec{T}^{b}$. The force $\vec{F}^{b}$ is explained by the Archimedes' Principle, which says that the buoyancy force always points upwards parallel to the local vertical and its magnitude is equal to the weight of the air volume displaced by the balloon. Therefore,

$$
\mathbf{F}_{\mathrm{G}}^{b}=V g \rho_{\mathrm{air}} \mathbf{e}_{3},
$$


where $V$ is the volume of the balloon (and of the gas as well), $g$ is the gravitational acceleration, and $\rho_{\text {air }}$ is the air density. Denote the magnitude of $\vec{F}^{b}$ by $F^{b} \triangleq V g \rho_{\text {air }}$.

On the other hand, the restoring torque $\vec{T}^{b}$ is an effort acting about the vehicle's CM, which appears as a consequence of the displacement $d$ between the balloon's CB and the vehicle's CM. Figure 3 illustrates the balloon-airframe connection. Assume that this connection is rigid. In this case, the $\mathrm{CB}$, which is placed at point $H$, is fixed w.r.t. $\mathcal{S}_{\mathrm{B}}$. From the illustration, one can immediately write

$$
\vec{T}^{b}=\left(d \hat{z}_{\mathrm{B}}\right) \times \vec{F}^{b}
$$

By representing equation (8) in $\mathcal{S}_{\mathrm{B}}$, we finally have

$$
\mathbf{T}_{\mathrm{B}}^{b}=d\left[\mathbf{e}_{3} \times\right] \mathbf{D}^{\mathrm{B} / \mathrm{G}} \mathbf{e}_{3} F^{b}
$$

where $\mathbf{D}^{\mathrm{B} / \mathrm{G}} \in \mathrm{SO}(3)$ is the attitude matrix of $\mathcal{S}_{\mathrm{B}}$ w.r.t. $\mathcal{S}_{\mathrm{G}}$.

\subsection{Translational Motion}

By invoking the Second Newton's Law considering all the vectors represented in $\mathcal{S}_{\mathrm{G}}$, one can immediately write 5

$$
\left(m^{t}+m^{h}\right) \ddot{\mathbf{r}}_{\mathrm{G}}^{\mathrm{B} / \mathrm{G}}=\mathbf{F}_{\mathrm{G}}^{g}+\mathbf{F}_{\mathrm{G}}^{b}+\mathbf{F}_{\mathrm{G}}^{c}+\mathbf{F}_{\mathrm{G}}^{d}
$$

where $m^{t}$ is the total mass of the vehicle without lifting gas and including the payload, $m^{h} \triangleq$ $\rho_{\text {helium }} V$ is the helium mass, with $\rho_{\text {helium }}$ representing the helium density, $\mathbf{r}_{\mathrm{G}}^{\mathrm{B} / \mathrm{G}} \in \mathbb{R}^{3}$ is the $\mathcal{S}_{\mathrm{G}}$ representation of the position of the vehicle's center of mass $B$ w.r.t. $G, \mathbf{F}_{\mathrm{G}}^{g}$ is the $\mathcal{S}_{\mathrm{G}}$ representation of the gravitational force, $\mathbf{F}_{\mathrm{G}}^{b}$ is the $\mathcal{S}_{\mathrm{G}}$ representation of the aerostatic lift force, $\mathbf{F}_{\mathrm{G}}^{c}$ is the $\mathcal{S}_{\mathrm{G}}$

representation of the control force, and $\mathbf{F}_{\mathrm{G}}^{d}$ is the $\mathcal{S}_{\mathrm{G}}$ representation of the (unknown) disturbance force. The force $\mathbf{F}_{\mathrm{G}}^{b}$ is given by equation (7), while $\mathbf{F}_{\mathrm{G}}^{g}$ and $\mathbf{F}_{\mathrm{G}}^{c}$ are modeled by

$$
\begin{aligned}
& \mathbf{F}_{\mathrm{G}}^{g}=-\left(m^{t}+m^{h}\right) g \mathbf{e}_{3}, \\
& \mathbf{F}_{\mathrm{G}}^{c}=F^{c}\left(\mathbf{D}^{\mathrm{B} / \mathrm{G}}\right)^{\mathrm{T}} \mathbf{e}_{3} .
\end{aligned}
$$


By replacing equations (11)-(12) into (10), one can obtain

$$
\ddot{\mathbf{r}}_{\mathrm{G}}^{\mathrm{B} / \mathrm{G}}=\frac{F^{c}}{m^{t}+m^{h}} \mathbf{n}_{\mathrm{G}}+\left(\frac{V g \rho_{\mathrm{air}}}{m^{t}+m^{h}}-g\right) \mathbf{e}_{3}+\frac{1}{m^{t}+m^{h}} \mathbf{F}_{\mathrm{G}}^{d},
$$

where $\mathbf{n}_{\mathrm{G}} \in \mathbb{R}^{3}$ is the transpose of the third line of $\mathrm{D}^{\mathrm{B} / \mathrm{G}}$, which corresponds to the $\mathcal{S}_{\mathrm{G}}$ representation of the unit (geometric) vector $\hat{z}_{\mathrm{B}}$.

\subsection{Rotational Motion}

The kinematic equation of the rotational motion of $\mathcal{S}_{\mathrm{B}}$ w.r.t. $\mathcal{S}_{\mathrm{G}}$ is given in $\mathrm{SO}(3)$ by

$$
\dot{\mathrm{D}}^{\mathrm{B} / \mathrm{G}}=-\left[\boldsymbol{\Omega}_{\mathrm{B}}^{\mathrm{B} / \mathrm{G}} \times\right] \mathbf{D}^{\mathrm{B} / \mathrm{G}}
$$

where $\Omega_{\mathrm{B}}^{\mathrm{B} / \mathrm{G}} \in \mathbb{R}^{3}$ is the $\mathcal{S}_{\mathrm{B}}$ representation of the vehicle's angular velocity w.r.t. $\mathcal{S}_{\mathrm{G}}$.

Assume that the vehicle has a rigid structure and consider $\mathcal{S}_{\mathrm{G}}$ as an inertial frame. Therefore, the Euler's Law gives

$$
\dot{\mathbf{H}}_{\mathrm{B}}+\left[\mathbf{\Omega}_{\mathrm{B}}^{\mathrm{B} / \mathrm{G}} \times\right] \mathbf{H}_{\mathrm{B}}=\mathbf{T}_{\mathrm{B}}^{c}+\mathbf{T}_{\mathrm{B}}^{b}+\mathbf{T}_{\mathrm{B}}^{d}
$$

where $\mathbf{H}_{\mathrm{B}}$ is the $\mathcal{S}_{\mathrm{B}}$ representation of the total angular momentum of the vehicle, $\mathbf{T}_{\mathrm{B}}^{c}$ is the $\mathcal{S}_{\mathrm{B}}$ representation of the control torque (see equation (5)), $\mathbf{T}_{\mathrm{B}}^{d}$ is the $\mathcal{S}_{\mathrm{B}}$ representation of the (unknown) disturbance torque, and $\mathbf{T}_{\mathrm{B}}^{b}$ is the $\mathcal{S}_{\mathrm{B}}$ representation of the balloon restoring torque (see equation (9)).

Considering the rotation of both the body and the propellers and noting that the latter rotates much faster, the total angular momentum $\mathbf{H}_{\mathrm{B}}$ can be written as

$$
\mathbf{H}_{\mathrm{B}}=\mathbf{J} \boldsymbol{\Omega}_{\mathrm{B}}^{\mathrm{B} / \mathrm{G}}+J^{r} \sum_{i=1}^{6}(-1)^{i} \omega_{i} \mathbf{e}_{3},
$$

where $\mathbf{J} \in \mathbb{R}^{3 \times 3}$ is the inertia matrix of the vehicle and $J^{r} \in \mathbb{R}$ is the moment of inertia of each rotor about $\hat{z}_{\mathrm{B}}$.

Therefore, by replacing equation (16) into equation (15), one can obtain the dynamic equation of the rotational motion of $\mathcal{S}_{\mathrm{B}}$ w.r.t. $\mathcal{S}_{\mathrm{G}}$ : 


$$
\begin{array}{r}
\dot{\boldsymbol{\Omega}}_{\mathrm{B}}^{\mathrm{B} / \mathrm{G}}=\mathbf{J}^{-1}\left[\left(\mathbf{J} \boldsymbol{\Omega}_{\mathrm{B}}^{\mathrm{B} / \mathrm{G}}+J^{r} \sum_{i=1}^{6}(-1)^{i} \omega_{i} \mathbf{e}_{3}\right) \times\right] \boldsymbol{\Omega}_{\mathrm{B}}^{\mathrm{B} / \mathrm{G}}-\mathbf{J}^{-1} J^{r} \sum_{i=1}^{6}(-1)^{i} \dot{\omega}_{i} \mathbf{e}_{3}+ \\
\mathbf{J}^{-1} \mathbf{T}_{\mathrm{B}}^{b}+\mathbf{J}^{-1} \mathbf{T}_{\mathrm{B}}^{c}+\mathbf{J}^{-1} \mathbf{T}_{\mathrm{B}}^{d} .
\end{array}
$$

\subsection{Added Mass}

The added-mass (also called virtual or apparent-mass) forces and torques are aerodynamic reaction efforts from a surrounding fluid on a body which tries to accelerate or decelerate inside it; note that the displaced mass of fluid is accelerated or decelerated together with the body interacting with it (see, e.g., $[18,19])$. This effect is only relevant if the mass of the displaced fluid is significant compared to the body's mass, which is generally the case for conventional or hybrid airships $[20,9,16]$. It is also worth noting that it appears even in low-speed and windless conditions.

From the Kirchhoff equation (for a body moving in an ideal fluid) one can show that the added-mass force and torque are, respectively, given by [18]

$$
\begin{aligned}
& {\left[\begin{array}{c}
\mathbf{F}_{\mathrm{B}}^{a} \\
\mathbf{T}_{\mathrm{B}}^{a}
\end{array}\right]=-\mathbf{M}^{a}\left[\begin{array}{c}
\dot{\mathbf{v}}_{\mathrm{B}}^{\mathrm{B} / \mathrm{G}} \\
\dot{\Omega}_{\mathrm{B}}^{\mathrm{B} / \mathrm{G}}
\end{array}\right]-} \\
& {\left[\begin{array}{c}
{\left[\boldsymbol{\Omega}_{\mathrm{B}}^{\mathrm{B} / \mathrm{G}} \times\right]\left(\mathbf{M}_{11} \mathbf{v}_{\mathrm{B}}^{\mathrm{B} / \mathrm{G}}+\mathrm{M}_{12} \boldsymbol{\Omega}_{\mathrm{B}}^{\mathrm{B} / \mathrm{G}}\right)} \\
{\left[\mathbf{v}_{\mathrm{B}}^{\mathrm{B} / \mathrm{G}} \times\right]\left(\mathbf{M}_{11} \mathbf{v}_{\mathrm{B}}^{\mathrm{B} / \mathrm{G}}+\mathrm{M}_{12} \boldsymbol{\Omega}_{\mathrm{B}}^{\mathrm{B} / \mathrm{G}}\right)+\left[\boldsymbol{\Omega}_{\mathrm{B}}^{\mathrm{B} / \mathrm{G}} \times\right]\left(\mathrm{M}_{21} \mathbf{v}_{\mathrm{B}}^{\mathrm{B} / \mathrm{G}}+\mathrm{M}_{22} \boldsymbol{\Omega}_{\mathrm{B}}^{\mathrm{B} / \mathrm{G}}\right)}
\end{array}\right],}
\end{aligned}
$$

where

$$
\mathbf{M}^{a} \triangleq\left[\begin{array}{ll}
\mathbf{M}_{11} & \mathbf{M}_{12} \\
\mathbf{M}_{21} & \mathbf{M}_{22}
\end{array}\right] \in \mathbb{R}^{6 \times 6},
$$

is the added mass in $\mathcal{S}_{\mathrm{B}}, \mathbf{M}_{i j} \in \mathbb{R}^{3 \times 3}, \forall i, j \in\{1,2\}$.

By writing the added-mass force as well as the vehicle's linear velocity in $\mathcal{S}_{\mathrm{G}}$, equation (18) 
yields

$$
\begin{aligned}
& \mathbf{F}_{\mathrm{G}}^{a}=-\mathbf{D}^{\mathrm{G} / \mathrm{B}} \mathbf{M}_{11} \mathbf{D}^{\mathrm{B} / \mathrm{G}} \dot{\mathbf{v}}_{\mathrm{G}}^{\mathrm{B} / \mathrm{G}}-\mathbf{D}^{\mathrm{G} / \mathrm{B}} \mathbf{M}_{12} \dot{\boldsymbol{\Omega}}_{\mathrm{B}}^{\mathrm{B} / \mathrm{G}}+\mathbf{D}^{\mathrm{G} / \mathrm{B}} \mathbf{M}_{11}\left[\boldsymbol{\Omega}_{\mathrm{B}}^{\mathrm{B} / \mathrm{G}} \times\right] \mathbf{D}^{\mathrm{B} / \mathrm{G}} \mathbf{v}_{\mathrm{G}}^{\mathrm{B} / \mathrm{G}} \\
& -\mathbf{D}^{\mathrm{G} / \mathrm{B}}\left[\boldsymbol{\Omega}_{\mathrm{B}}^{\mathrm{B} / \mathrm{G}} \times\right]\left(\mathbf{M}_{11} \mathbf{D}^{\mathrm{B} / \mathrm{G}} \mathbf{v}_{\mathrm{G}}^{\mathrm{B} / \mathrm{G}}+\mathbf{M}_{12} \boldsymbol{\Omega}_{\mathrm{B}}^{\mathrm{B} / \mathrm{G}}\right), \\
& \mathbf{T}_{\mathrm{B}}^{a}=-\mathbf{M}_{21} \mathbf{D}^{\mathrm{B} / \mathrm{G}} \dot{\mathbf{v}}_{\mathrm{G}}^{\mathrm{B} / \mathrm{G}}-\mathbf{M}_{22} \dot{\Omega}_{\mathrm{B}}^{\mathrm{B} / \mathrm{G}}+\mathbf{M}_{21}\left[\boldsymbol{\Omega}_{\mathrm{B}}^{\mathrm{B} / \mathrm{G}} \times\right] \mathbf{D}^{\mathrm{B} / \mathrm{G}} \mathbf{v}_{\mathrm{G}}^{\mathrm{B} / \mathrm{G}} \\
& -\left[\left(\mathbf{D}^{\mathrm{B} / \mathrm{G}} \mathbf{v}_{\mathrm{G}}^{\mathrm{B} / \mathrm{G}}\right) \times\right]\left(\mathbf{M}_{11} \mathbf{D}^{\mathrm{B} / \mathrm{G}} \mathbf{v}_{\mathrm{G}}^{\mathrm{B} / \mathrm{G}}+\mathbf{M}_{12} \boldsymbol{\Omega}_{\mathrm{B}}^{\mathrm{B} / \mathrm{G}}\right) \\
& -\left[\Omega_{\mathrm{B}}^{\mathrm{B} / \mathrm{G}} \times\right]\left(\mathrm{M}_{21} \mathrm{D}^{\mathrm{B} / \mathrm{G}} \mathbf{v}_{\mathrm{G}}^{\mathrm{B} / \mathrm{G}}+\mathrm{M}_{22} \Omega_{\mathrm{B}}^{\mathrm{B} / \mathrm{G}}\right) .
\end{aligned}
$$

Before computing $\mathbf{M}^{a}$ for the oblate-spheroid balloon under consideration, it is convenient to obtain the added mass $\check{\mathbf{M}}^{a} \triangleq \operatorname{diag}\left(\check{\mathbf{M}}_{11}, \check{\mathbf{M}}_{22}\right)$ with respect to a CCS which is parallel to $\mathcal{S}_{\mathrm{B}}$ and has its origin at $\mathrm{CB}$, using the procedure of [21], resulting in

$$
\check{\mathbf{M}}_{11}=\left[\begin{array}{ccc}
\check{m} & 0 & 0 \\
0 & \check{m} & 0 \\
0 & 0 & \check{m}_{3}
\end{array}\right], \text { and } \check{\mathbf{M}}_{22}=\left[\begin{array}{ccc}
\check{J} & 0 & 0 \\
0 & \check{J} & 0 \\
0 & 0 & 0
\end{array}\right]
$$

where 


$$
\begin{aligned}
\check{m} & \triangleq \frac{4 \pi}{3} a^{2} b \rho_{\text {air }} \frac{\alpha_{0}}{2-\alpha_{0}}, \\
\check{m}_{3} & \triangleq \frac{4 \pi}{3} a^{2} b \rho_{\text {air }} \frac{\beta_{0}}{2-\beta_{0}}, \\
\check{J} & \triangleq \frac{4 \pi}{15} a^{2} b \rho_{\text {air }}\left(a^{2}+b^{2}\right) \frac{\beta_{0}-\alpha_{0}}{2}, \\
\alpha_{0} & \triangleq \frac{\sqrt{1-e^{2}}}{e^{3}}\left(\sin ^{-1} e-e \sqrt{1-e^{2}}\right), \\
\beta_{0} & \triangleq \frac{2 \sqrt{1-e^{2}}}{e^{3}}\left(\frac{e}{\sqrt{1-e^{2}}}-\sin ^{-1} e\right), \\
e & \triangleq \sqrt{\frac{a^{2}-b^{2}}{a^{2}}} .
\end{aligned}
$$

Finally, to obtain $\mathbf{M}^{a}$ (in $\mathcal{S}_{\mathrm{B}}$ ), one can use the coordinate transformation (see [22], p.27)

$$
\mathbf{M}^{a}=\mathbf{U}^{\mathrm{T}} \check{\mathbf{M}}^{a} \mathbf{U}
$$

with

$$
\mathbf{U} \triangleq\left[\begin{array}{cc}
\mathbf{I}_{3} & -d\left[\mathbf{e}_{3} \times\right] \\
\mathbf{0}_{3 \times 3} & \mathbf{I}_{3}
\end{array}\right]
$$

Therefore, to account for the added mass, the new external inputs represented by $\mathbf{F}_{\mathrm{G}}^{a}$ and $\mathbf{T}_{\mathrm{B}}^{a}$ have to be considered in equations (13) and (17), respectively.

\section{Flight Control System}

A hierarchical control strategy is adopted here, as illustrated in Figure 4. In this strategy, the flight control is realized by two nested control loops, where the inner loop is responsible for the attitude control, while the outer loop performs position control. The position controller receives an external position command $\overline{\mathbf{r}}_{\mathrm{G}}^{\mathrm{B} / \mathrm{G}} \in \mathbb{R}^{3}$ as well as feedbacks of the vehicle position $\mathbf{r}_{\mathrm{G}}^{\mathrm{B} / \mathrm{G}}$ and velocity $\mathbf{v}_{\mathrm{G}}^{\mathrm{B} / \mathrm{G}}$. On the other hand, it produces the $\mathcal{S}_{\mathrm{G}}$ representation of the thrust command vector $\overline{\mathbf{F}}_{\mathrm{G}}^{c} \triangleq \bar{F}^{c} \overline{\mathbf{n}}_{\mathrm{G}} \in \mathbb{R}^{3}$, where $\bar{F}^{c} \in \mathbb{R}$ is the total thrust magnitude command and $\overline{\mathbf{n}}_{\mathrm{G}} \in \mathbb{R}^{3}$ (a unit vector) can be seen as a two-DOF attitude command that, together with the external 
Flight Control System

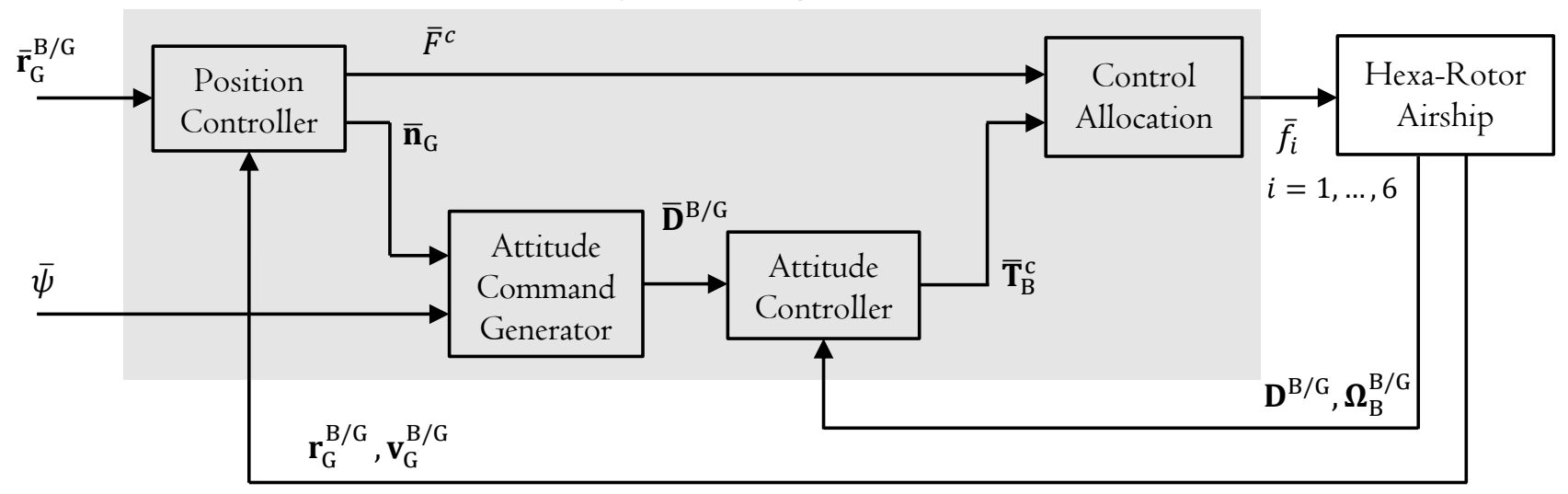

Figure 4: Architecture of the hexa-rotor airship control system.

heading command $\bar{\psi} \in \mathbb{R}$, composes the three-DOF command input $\overline{\mathbf{D}}^{\mathrm{B} / \mathrm{G}} \in \mathrm{SO}(3)$ of the attitude controller. The latter also receives feedback of the vehicle's attitude $\mathbf{D}^{\mathrm{B} / \mathrm{G}}$ and angular velocity $\boldsymbol{\Omega}_{\mathrm{B}}^{\mathrm{B} / \mathrm{G}}$, and computes the torque command $\overline{\mathbf{T}}_{\mathrm{B}}^{c} \in \mathbb{R}^{3}$. The control allocation block is responsible for generating the individual thrust commands $\bar{f}_{i} \in \mathbb{R}_{\geq 0}, i=1, \ldots, 6$, from $\overline{\mathbf{T}}_{\mathrm{B}}^{c}$ and $\bar{F}^{c}$.

This architecture is based on the assumption that there is a time-scale separation between the (slower) closed-loop translational and (faster) rotational vehicle dynamics [12]. This assumption is ensured in practice by tuning the attitude control loop to converge much faster than the position control loop. Under such assumptions, when designing the position control law, one can assume that the actual attitude $\mathbf{D}^{\mathrm{B} / \mathrm{G}}$ converges to the corresponding command $\overline{\mathbf{D}}^{\mathrm{B} / \mathrm{G}}$ instantaneously, i.e., $\mathbf{D}^{\mathrm{B} / \mathrm{G}} \equiv \overline{\mathbf{D}}^{\mathrm{B} / \mathrm{G}}$. On the other hand, when designing the attitude control law, the attitude command $\overline{\mathbf{D}}^{\mathrm{B} / \mathrm{G}}$ is assumed to be constant, or equivalently, the angular velocity command $\overline{\boldsymbol{\Omega}}_{\mathrm{B}}^{\mathrm{B} / \mathrm{G}} \in \mathbb{R}^{3}$ is assumed to be zero. On the basis of these conditions, the position and attitude control laws can be designed separately.

\subsection{Position Controller}

First, the design model is obtained from equation (13), by assuming that: 1) the disturbance force $\mathbf{F}_{\mathrm{G}}^{d}$ is negligible, 2) the actual control force magnitude $F^{c}$ is identical to the corresponding 
command $\bar{F}^{c}$, and 3) $\mathrm{D}^{\mathrm{B} / \mathrm{G}}=\overline{\mathrm{D}}^{\mathrm{B} / \mathrm{G}}$ (time-scale separation). The resulting design model is

$$
\ddot{\mathbf{r}}_{\mathrm{G}}^{\mathrm{B} / \mathrm{G}}=\frac{1}{m^{t}+m^{h}} \overline{\mathbf{F}}_{\mathrm{G}}^{c}+\left(\frac{V g \rho_{\mathrm{air}}}{m^{t}+m^{h}}-g\right) \mathbf{e}_{3} .
$$

Suppose that $\overline{\mathbf{F}}_{\mathrm{G}}^{c}$ is bounded within a parallelepipedal set from $\mathbf{F}^{\min } \triangleq\left[F_{1}^{\min } F_{2}^{\min } F_{3}^{\min }\right]^{\mathrm{T}} \in \mathbb{R}^{3}$ to $\mathbf{F}^{\max } \triangleq\left[F_{1}^{\max } F_{2}^{\max } F_{3}^{\max }\right]^{\mathrm{T}} \in \mathbb{R}^{3}$. The position controller proposed here is the saturated law

$$
\overline{\mathbf{F}}_{\mathrm{G}}^{c}=\boldsymbol{\sigma}_{\left[\mathbf{F}^{\min }, \mathbf{F}^{\max ]}\right.}\left(\gamma^{p}\right),
$$

where $\gamma^{p} \equiv\left[\begin{array}{lll}\gamma_{1}^{p} & \gamma_{2}^{p} & \gamma_{3}^{p}\end{array}\right]^{\mathrm{T}} \in \mathbb{R}^{3}$ is given by

$$
\gamma^{p} \triangleq\left(\left(m^{t}+m^{h}\right) g-V g \rho_{\text {air }}\right) \mathbf{e}_{3}+\left(m^{t}+m^{h}\right) \mathbf{K}_{1}\left(\overline{\mathbf{r}}_{\mathrm{G}}^{\mathrm{B} / \mathrm{G}}-\mathbf{r}_{\mathrm{G}}^{\mathrm{B} / \mathrm{G}}\right)-\left(m^{t}+m^{h}\right) \mathbf{K}_{2} \dot{\mathbf{r}}_{\mathrm{G}}^{\mathrm{B} / \mathrm{G}}
$$

with the matrices $\mathbf{K}_{1}, \mathbf{K}_{2} \in \mathbb{R}^{3 \times 3}$ representing the controller gains (assumed to be diagonal), and

$$
\begin{aligned}
& \boldsymbol{\sigma}_{\left[\mathbf{F}^{\min }, \mathbf{F}^{\max }\right]}\left(\gamma^{p}\right) \triangleq {\left[\begin{array}{c}
\sigma_{\left[F_{1}^{\min }, F_{1}^{\max }\right]}\left(\gamma_{1}^{p}\right) \\
\sigma_{\left[F_{2}^{\min }, F_{2}^{\max }\right]}\left(\gamma_{2}^{p}\right) \\
\sigma_{\left[F_{3}^{\min }, F_{3}^{\max }\right]}\left(\gamma_{3}^{p}\right)
\end{array}\right], } \\
& \sigma_{\left[F_{l}^{\max }, F_{l}^{\max }\right]}\left(\gamma_{l}^{p}\right) \triangleq\left\{\begin{array}{cc}
F_{l}^{\min }, & \gamma_{l}^{p}<F_{l}^{\min } \\
\gamma_{l}^{p}, & \gamma_{l}^{p} \in\left[F_{l}^{\min }, F_{l}^{\max }\right], l=1,2,3 . \\
F_{l}^{\max }, & \gamma_{l}^{p}>F_{l}^{\max }
\end{array}\right.
\end{aligned}
$$

Note that the proposed controller (32)-(33) is such that, if no saturation is active, it cancels the second term on the right-hand side of equation (33), remaining a double-integrator closedloop dynamics controlled by the proportional-derivative actions appearing in the last two terms of equation (33). In saturation-free conditions, it is straightforward to show asymptotic stability of the proposed translational control loop using linear time-invariant control methods. Subsection 3.5 presents a stability analysis considering the saturation.

By constraining the control input $\overline{\mathbf{F}}_{\mathrm{G}}^{c}$ to keep inside a parallelepipedal set, one can deal with 


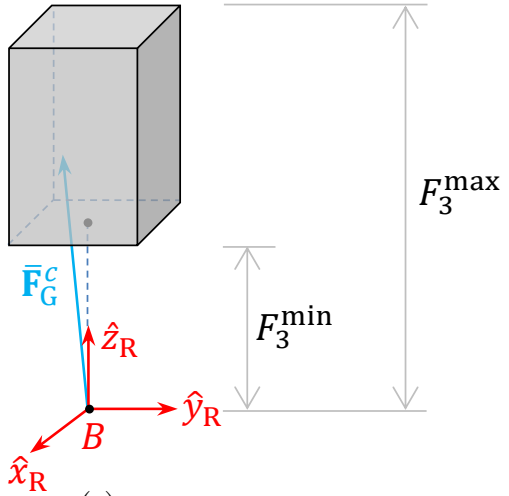

(a)

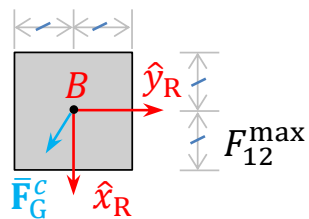

(b)

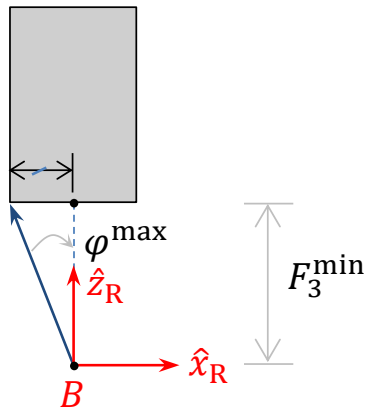

(c)

Figure 5: Constraint set of the position control input. (a) Perspective view showing the control force command inside the parallelepipedal set. (b) Superior view showing the bounds $F_{1}^{\max }=F_{1}^{\max }=F_{12}^{\max }$. (c) Lateral view showing the maximum inclination angle $\varphi^{\max }$.

bounds on its magnitude $\bar{F}^{c}$ as well as on its inclination angle w.r.t. the local vertical. To illustrate it, define a reference CCS $\mathcal{S}_{\mathrm{R}} \triangleq\left\{B ; \hat{x}_{\mathrm{R}}, \hat{y}_{\mathrm{R}}, \hat{z}_{\mathrm{R}}\right\}$ with origin at $B$ and axes parallel to the $\mathcal{S}_{\mathrm{G}}$ ones, as depicted in Figure 5. From the geometry of the constraint set, one can see that $F_{1}^{\min }=-F_{1}^{\max }$, $F_{2}^{\min }=-F_{2}^{\max }$, and $F_{1}^{\max }=F_{2}^{\max }=F_{12}^{\max }=F_{3}^{\min } \tan \varphi^{\max }$, where $\varphi^{\max }$ is the maximum allowed inclination angle of $\overline{\mathbf{F}}_{\mathrm{G}}^{c}$ w.r.t. $\hat{z}_{\mathrm{R}}$.

\subsection{Attitude Controller}

Here the design model is obtained from equation (17) by: 1) neglecting the disturbance torque $\mathbf{T}_{\mathrm{B}}^{d}$; 2) replacing the actual control torque $\mathbf{T}_{\mathrm{B}}^{c}$ by the corresponding command $\overline{\mathbf{T}}_{\mathrm{B}}^{c} ; 3$ ) considering that the rotor dynamics are so fast that one can assume $\omega_{i}=\bar{\omega}_{i}, \forall i$; and 4) assuming that $\dot{\omega}_{i} \approx 0$, $\forall i$. The resulting model is

$$
\dot{\boldsymbol{\Omega}}_{\mathrm{B}}^{\mathrm{B} / \mathrm{G}}=\mathbf{J}^{-1}\left[\left(\mathbf{J} \boldsymbol{\Omega}_{\mathrm{B}}^{\mathrm{B} / \mathrm{G}}+J^{r} \sum_{i=1}^{6}(-1)^{i} \bar{\omega}_{i} \mathbf{e}_{3}\right) \times\right] \boldsymbol{\Omega}_{\mathrm{B}}^{\mathrm{B} / \mathrm{G}}+d F^{b} \mathbf{J}^{-1}\left[\mathbf{e}_{3} \times\right] \mathbf{D}^{\mathrm{B} / \mathrm{G}} \mathbf{e}_{3}+\mathbf{J}^{-1} \overline{\mathbf{T}}_{\mathrm{B}}^{c} .
$$

Suppose that the torque command $\overline{\mathbf{T}}_{\mathrm{B}}^{c}$ must be bounded from $-\mathbf{T}^{\max } \in \mathbb{R}^{3}$ to $\mathbf{T}^{\max } \triangleq$ $\left[T_{1}^{\max } T_{2}^{\max } T_{3}^{\max }\right]^{\mathrm{T}}$. The attitude controller proposed here is the saturated law

$$
\overline{\mathbf{T}}_{\mathrm{B}}^{c}=\boldsymbol{\sigma}_{\left[-\mathbf{T}^{\max }, \mathbf{T}^{\max }\right]}\left(\gamma^{a}\right),
$$


where

$$
\begin{aligned}
\gamma^{a} \triangleq-d F^{b}\left[\mathbf{e}_{3} \times\right] \mathbf{D}^{\mathrm{B} / \mathrm{G}} \mathbf{e}_{3}+\left[\boldsymbol{\Omega}_{\mathrm{B}}^{\mathrm{B} / \mathrm{G}} \times\right] \mathbf{J} \boldsymbol{\Omega}_{\mathrm{B}}^{\mathrm{B} / \mathrm{G}}+J^{r}\left[\boldsymbol{\Omega}_{\mathrm{B}}^{\mathrm{B} / \mathrm{G}} \times\right] \mathbf{e}_{3} \sum_{i=1}^{6}(-1)^{i} \bar{\omega}_{i} \\
\quad-\mathbf{J K}_{3} \boldsymbol{\varepsilon}-\mathbf{J K}_{4} \boldsymbol{\Omega}_{\mathrm{B} / \mathrm{G}}^{\mathrm{B}},
\end{aligned}
$$

with $\varepsilon \in \mathbb{R}^{3}$ containing the Euler angles (in the 1-2-3 sequence) corresponding to the attitude

control error $\tilde{\mathbf{D}}=\mathbf{D}^{\mathrm{B} / \mathrm{G}}\left(\overline{\mathbf{D}}^{\mathrm{B} / \mathrm{G}}\right)^{\mathrm{T}}, \mathbf{K}_{3}, \mathbf{K}_{4} \in \mathbb{R}^{3 \times 3}$ are controller (diagonal-matrix) gains, and the saturation function $\boldsymbol{\sigma}_{\left[-\mathbf{T}^{\max }, \mathbf{T}^{\max }\right]}($.$) is as defined in equations (34)-(35).$

One can note that the proposed attitude control law (37)-(38) is such that, if no saturation is active, it cancels the first three terms on the right-hand side of equation (38), remaining a feedback-linearized closed-loop dynamic model controlled by the proportional-derivative actions appearing in the last two terms of (38). Subsection 3.5 presents a stability analysis.

\subsection{Attitude Command Generation}

In order to generate the three-DOF attitude command $\overline{\mathbf{D}}^{\mathrm{B} / \mathrm{R}}$, note that, by definition, its third line is the transpose of $\overline{\mathbf{n}}_{\mathrm{G}}$. Then, consider the formula to convert from Euler angles (in the 1-2-3 sequence) to the attitude matrix (see [23], p. 52):

$$
\overline{\mathbf{D}}^{\mathrm{B} / \mathrm{R}}=\left[\begin{array}{ccc}
* & * & * \\
* & * & * \\
\mathrm{~s} \bar{\theta} & -\mathrm{c} \bar{\theta} \mathrm{s} \bar{\phi} & \mathrm{c} \bar{\theta} \mathrm{c} \bar{\phi}
\end{array}\right]
$$

where s and c stands for sin and cos, respectively, and compute $\bar{\phi}$ and $\bar{\theta}$ from

$$
\begin{aligned}
& \bar{\phi}=-\operatorname{atan} \mathbf{e}_{2}^{\mathrm{T}} \overline{\mathbf{n}}_{\mathrm{G}} / \mathbf{e}_{3}^{\mathrm{T}} \overline{\mathbf{n}}_{\mathrm{G}}, \\
& \bar{\theta}=\operatorname{asin} \mathbf{e}_{1}^{\mathrm{T}} \overline{\mathbf{n}}_{\mathrm{G}} .
\end{aligned}
$$


Finally, considering the external heading command $\bar{\psi}$, one can compute

$$
\overline{\mathbf{D}}^{\mathrm{B} / \mathrm{R}}=\left[\begin{array}{ccc}
\mathrm{c} \bar{\psi} \mathrm{c} \bar{\theta} & \mathrm{c} \bar{\psi} \mathrm{s} \bar{\theta} \mathrm{s} \bar{\phi}+\mathrm{s} \bar{\psi} \mathrm{c} \bar{\phi} & -\mathrm{c} \bar{\psi} \mathrm{s} \bar{\theta} \mathrm{c} \bar{\phi}+\mathrm{s} \bar{\psi} \mathrm{s} \bar{\phi} \\
-\mathrm{s} \psi \mathrm{c} \bar{\theta} & -\mathrm{s} \bar{\psi} \mathrm{s} \bar{\theta} \mathrm{s} \bar{\phi}+\mathrm{c} \bar{\psi} \mathrm{c} \bar{\phi} & \mathrm{s} \bar{\psi} \mathrm{s} \bar{\theta} \mathrm{c} \bar{\phi}+\mathrm{c} \bar{\psi} \mathrm{s} \bar{\phi} \\
\mathrm{s} \bar{\theta} & -\mathrm{c} \bar{\theta} \mathrm{s} \bar{\phi} & \mathrm{c} \bar{\theta} \mathrm{c} \bar{\phi}
\end{array}\right]
$$

\subsection{Control Allocation}

Consider equation (5) again; it relates the effective resultant efforts $F^{c}$ and $\mathbf{T}_{\mathrm{B}}^{c}$ with the six individual rotor forces (stored in $\mathbf{f}$ ). We can consider that the respective effort commands are interrelated in the same way, i.e.,

$$
\left[\begin{array}{l}
\bar{F}^{c} \\
\overline{\mathbf{T}}_{\mathrm{B}}^{c}
\end{array}\right]=\overline{\boldsymbol{\Gamma}},
$$

where $\overline{\mathbf{f}} \triangleq\left[\begin{array}{llll}\bar{f}_{1} & \bar{f}_{2} & \ldots & \bar{f}_{6}\end{array}\right]^{\mathrm{T}}$.

The control allocation pursued here is to find a solution $\overline{\mathbf{f}}$ to the system of linear equations (43). Since this is an undetermined system (with infinitely many solutions), we choose here the

one that minimizes $\|\overline{\mathbf{f}}\|^{2}$, considering (43) as a set of equality constraints. The explicit solution to such a problem is well known; by using Lagrange multipliers, it can be found to be

$$
\overline{\mathbf{f}}=\boldsymbol{\Gamma}^{\mathrm{T}}\left(\boldsymbol{\Gamma} \boldsymbol{\Gamma}^{\mathrm{T}}\right)^{-1}\left[\begin{array}{c}
\bar{F}^{c} \\
\overline{\mathbf{T}}_{\mathrm{B}}^{c}
\end{array}\right] .
$$

The above control allocation can be seen as an energy-efficient solution, since it minimizes the squared norm of $\overline{\mathbf{f}}$. Moreover, considering that the commands $\bar{F}^{c}$ and $\overline{\mathbf{T}}_{\mathrm{B}}^{c}$ belong to the admissible set of the above optimization problem (meaning that the problem has at least one solution), the resulting control allocation is exact, i.e., it satisfies equation (43).

\subsection{Stability Analysis}

Still considering the time-scale separation assumption, this subsection presents stability proofs for both the position and attitude control loops. 


\subsubsection{Position control}

Denote the admissible-force set by

$$
\mathcal{F} \triangleq\left\{\mathbf{F} \in \mathbb{R}^{3}: \mathbf{F}^{\min } \leq \mathbf{F} \leq \mathbf{F}^{\max }\right\}
$$

and define the tracking error $\tilde{\mathbf{r}} \triangleq \mathbf{r}_{\mathrm{G}}^{\mathrm{B} / \mathrm{G}}-\overline{\mathbf{r}}_{\mathrm{G}}^{\mathrm{B} / \mathrm{G}}$, the viable set

$$
\mathcal{L}_{p} \triangleq\left\{(\tilde{\mathbf{r}}, \dot{\tilde{\mathbf{r}}}) \in \mathbb{R}^{6}: \gamma^{p} \in \mathcal{F}\right\}
$$

as well as the maximal 0-centered Euclidean ball inside $\mathcal{L}_{p}$

$$
\mathcal{L}_{p}^{*} \triangleq \max _{\lambda} \mathcal{B}_{\lambda} \text { s.t. } \mathcal{B}_{\lambda} \subseteq \mathcal{L}_{p}
$$

where $\mathcal{B}_{\lambda} \triangleq\left\{\mathbf{x} \in \mathbb{R}^{6}:\|\mathbf{x}\| \leq \lambda\right\}$.

The following theorem gives a stability result for the closed-loop system (31)-(33).

Theorem 1. Consider the closed-loop system (31)-(33). Assume that the position command $\overline{\mathbf{r}}_{\mathrm{G}}^{\mathrm{B} / \mathrm{G}}$ is such that $\ddot{\mathbf{r}}_{\mathrm{G}}^{\mathrm{B} / \mathrm{G}}(t)=0, \forall t \in \mathbb{R}_{\geq 0}$. In this context, if the initial state $\left(\mathbf{r}_{\mathrm{G}}^{\mathrm{B} / \mathrm{G}}(0), \dot{\mathbf{r}}_{\mathrm{G}}^{\mathrm{B} / \mathrm{G}}(0)\right) \in$ $\mathcal{L}_{p}^{*} \oplus\left(\overline{\mathbf{r}}_{\mathrm{G}}^{\mathrm{B} / \mathrm{G}}(0), \dot{\mathbf{r}}_{\mathrm{G}}^{\mathrm{B} / \mathrm{G}}(0)\right)$, then $\mathbf{r}_{\mathrm{G}}^{\mathrm{B} / \mathrm{G}}$ converges exponentially to $\overline{\mathbf{r}}_{\mathrm{G}}^{\mathrm{B} / \mathrm{G}}$ and $\left(\mathbf{r}_{\mathrm{G}}^{\mathrm{B} / \mathrm{G}}(t), \dot{\mathbf{r}}_{\mathrm{G}}^{\mathrm{B} / \mathrm{G}}(t)\right) \in \mathcal{L}_{p}^{*} \oplus$ $\left(\overline{\mathbf{r}}_{\mathrm{G}}^{\mathrm{B} / \mathrm{G}}(t), \dot{\mathbf{r}}_{\mathrm{G}}^{\mathrm{B} / \mathrm{G}}(t)\right), \forall t \in \mathbb{R}_{>0}$.

Proof. Under the condition that $\gamma^{p} \in \mathcal{F}$ or, equivalently, $(\tilde{\mathbf{r}}, \dot{\tilde{\mathbf{r}}}) \in \mathcal{L}_{p}$, the closed-loop translational dynamics represented by equations (31)-(33) can be re-modeled in terms of $\tilde{\mathbf{r}}$ and its time derivatives, resulting in

$$
\ddot{\tilde{\mathbf{r}}}+\mathbf{K}_{2} \dot{\tilde{\mathbf{r}}}+\mathbf{K}_{1} \tilde{\mathbf{r}}=-\ddot{\mathbf{r}}_{\mathrm{G}}^{\mathrm{B} / \mathrm{G}} .
$$

Since, by assumption, $\ddot{\mathbf{r}}_{\mathrm{G}}^{\mathrm{B} / \mathrm{G}}(t)=0, \forall t \in \mathbb{R}_{\geq 0}$ and the gain matrices $\mathbf{K}_{3}$ and $\mathbf{K}_{4}$ are positive-definite and diagonal, from the linear control theory, one see that the state $(\tilde{\mathbf{r}}, \dot{\tilde{\mathbf{r}}})=\left(\mathbf{0}_{3 \times 1}, \mathbf{0}_{3 \times 1}\right)$ of system (48), without considering the control saturation, is globally exponentially stable. This implies that, for any $\lambda \in \mathbb{R}_{>0}$, if $(\tilde{\mathbf{r}}(0), \dot{\tilde{\mathbf{r}}}(0)) \in \mathcal{B}_{\lambda}$, then $(\tilde{\mathbf{r}}(t), \dot{\tilde{\mathbf{r}}}(t)) \in \mathcal{B}_{\lambda}, \forall t \in \mathbb{R}_{>0}$, which means that $\mathcal{B}_{\lambda}$ is an invariant set of system (48).

In particular, consider the ball $\mathcal{L}_{p}^{*}$ defined in (47). Since, by construction, $\mathcal{L}_{p}^{*} \subseteq \mathcal{L}_{p}$, it holds that 
$\gamma^{p} \in \mathcal{F}$, from the above argumentation, one can see that $\mathcal{L}_{p}^{*}$ is an invariant set of system (31)-(33), which, in this case, behaves as (48) (with $\ddot{\overline{\mathbf{r}}}_{\mathrm{G}}^{\mathrm{B}} \mathrm{G}(t)=0, \forall t \in \mathbb{R}_{\geq 0}$ ). Therefore, if $(\tilde{\mathbf{r}}(0), \dot{\tilde{\mathbf{r}}}(0)) \in \mathcal{L}_{p}^{*}$, then $(\tilde{\mathbf{r}}(t), \dot{\tilde{\mathbf{r}}}(t)) \in \mathcal{L}_{p}^{*}, \forall t \in \mathbb{R}_{>0}$ and $\tilde{\mathbf{r}}$ converges to $\mathbf{0}_{3 \times 1}$ exponentially. From the definition of $\tilde{\mathbf{r}}$, this is equivalent to say that $\left(\mathbf{r}_{\mathrm{G}}^{\mathrm{B} / \mathrm{G}}(t), \dot{\mathbf{r}}_{\mathrm{G}}^{\mathrm{B} / \mathrm{G}}(t)\right) \in \mathcal{L}_{p}^{*} \oplus\left(\overline{\mathbf{r}}_{\mathrm{G}}^{\mathrm{B} / \mathrm{G}}(t), \dot{\mathbf{r}}_{\mathrm{G}}^{\mathrm{B} / \mathrm{G}}(t)\right), \forall t \in \mathbb{R}_{\geq 0}$ and $\mathbf{r}_{\mathrm{G}}^{\mathrm{B} / \mathrm{G}}$ converges to $\overline{\mathbf{r}}_{\mathrm{G}}^{\mathrm{B} / \mathrm{G}}$ exponentially, thus completing the proof.

\subsubsection{Attitude Control}

Denote the admissible-torque set

$$
\mathcal{T} \triangleq\left\{\mathbf{T} \in \mathbb{R}^{3}:-\mathbf{T}^{\max } \leq \mathbf{T} \leq \mathbf{T}^{\max }\right\}
$$

and define the corresponding viable set

$$
\mathcal{L}_{a} \triangleq\left\{\left(\varepsilon, \Omega_{\mathrm{B}}^{\mathrm{B} / \mathrm{G}}\right) \in \mathbb{R}^{6}: \gamma^{a} \in \mathcal{T}\right\}
$$

as well as the maximal 0-centered Euclidean ball inside $\mathcal{L}_{a}$

$$
\mathcal{L}_{a}^{*} \triangleq \max _{\lambda} \mathcal{B}_{\lambda} \text { s.t. } \mathcal{B}_{\lambda} \subseteq \mathcal{L}_{a}
$$

From the assumption that $\overline{\mathbf{D}}^{\mathrm{B} / \mathrm{G}}(t)$ is constant (time-scale separation), it follows that $\bar{\Omega}_{\mathrm{B}}^{\mathrm{B} / \mathrm{G}}(t)=$ 0. In this case, the attitude error kinematics can be described by (see [23], p. 73)

$$
\dot{\varepsilon}=\mathrm{A}(\varepsilon) \Omega_{\mathrm{B}}^{\mathrm{B} / \mathrm{G}}
$$

where

$$
\mathbf{A}(\boldsymbol{\varepsilon}) \triangleq\left[\begin{array}{ccc}
\mathrm{c} \varepsilon_{3} / \mathrm{c} \varepsilon_{2} & -\mathrm{s} \varepsilon_{3} / \mathrm{c} \varepsilon_{2} & 0 \\
\mathrm{~s} \varepsilon_{3} & \mathrm{c} \varepsilon_{3} & 0 \\
-\mathrm{c} \varepsilon_{3} \mathrm{~s} \varepsilon_{2} / \mathrm{c} \varepsilon_{2} & \mathrm{~s} \varepsilon_{3} \mathrm{~s} \varepsilon_{2} / \mathrm{c} \varepsilon_{2} & 1
\end{array}\right]
$$

with $\varepsilon_{i}, i=1,2,3$, denoting the components of $\varepsilon$.

In this section, we assume that $\varepsilon$ has small components. Such small-angle approximation is 
reasonable, since $\varepsilon$ represents an attitude error rather than the total attitude itself and, from the time-scale separation assumption, $\mathbf{D}^{\mathrm{B} / \mathrm{G}}$ is supposed to rapidly converge to $\overline{\mathbf{D}}^{\mathrm{B} / \mathrm{G}}$ (which means that $\varepsilon$ rapidly converges to zero). In this case, equation (52) can be approximated by

$$
\dot{\varepsilon}=\Omega_{\mathrm{B}}^{\mathrm{B} / \mathrm{G}} .
$$

The following theorem gives a stability result for the closed-loop system (52), (37)-(38).

Theorem 2. Consider the closed-loop system (52), (37)-(38). Assume that $\overline{\mathbf{D}}^{\mathrm{B} / \mathrm{G}}(t)$ is constant at any $t \in \mathbb{R}_{\geq 0}$ (time-scale separation) and that $\varepsilon$ is so small that its kinematics can be described by (54). In this context, if the initial state $\left(\varepsilon(0), \Omega_{\mathrm{B}}^{\mathrm{B} / \mathrm{G}}(0)\right) \in \mathcal{L}_{a}^{*}$, then $\mathbf{D}^{\mathrm{B} / \mathrm{G}}$ converges exponentially to $\overline{\mathbf{D}}^{\mathrm{B} / \mathrm{G}}$ and $\left(\varepsilon(t), \Omega_{\mathrm{B}}^{\mathrm{B} / \mathrm{G}}(t)\right) \in \mathcal{L}_{a}^{*}, \forall t \in \mathbb{R}_{>0}$.

Proof. Under the condition that $\gamma^{a} \in \mathcal{T}$ or, equivalently, $\left(\varepsilon, \Omega_{\mathrm{B}}^{\mathrm{B} / \mathrm{G}}\right) \in \mathcal{L}_{a}$, and considering the smallangle kinematics described by (54), the closed-loop rotational dynamics represented by equations (54),(37)-(38) can be re-modeled in terms of $\varepsilon$ and its time derivatives, resulting in

$$
\ddot{\varepsilon}+\mathbf{K}_{4} \dot{\varepsilon}+\mathbf{K}_{3} \varepsilon=\mathbf{0} .
$$

Since the gain matrices $\mathbf{K}_{3}$ and $\mathbf{K}_{4}$ are positive-definite and diagonal, from the linear control theory, one see that the state $(\varepsilon, \dot{\varepsilon})=\left(\mathbf{0}_{3 \times 1}, \mathbf{0}_{3 \times 1}\right)$ of system $(55)$, without considering the control saturation, is globally exponentially stable. This implies that, for any $\lambda \in \mathbb{R}_{>0}$, if $(\varepsilon(0), \dot{\varepsilon}(0)) \in \mathcal{B}_{\lambda}$, then $(\varepsilon(t), \dot{\varepsilon}(t)) \in \mathcal{B}_{\lambda}, \forall t \in \mathbb{R}_{>0}$, which means that $\mathcal{B}_{\lambda}$ is an invariant set of system (55).

In particular, consider the ball $\mathcal{L}_{a}^{*}$ defined in (51). Since, by construction, $\mathcal{L}_{a}^{*} \subseteq \mathcal{L}_{a}$, it holds that $\gamma^{a} \in \mathcal{T}$, from the above argumentation and assumptions, one can see that $\mathcal{L}_{a}^{*}$ is an invariant set of system (54), (37)-(38), which, in this case, behaves as (55). Therefore, if $(\varepsilon(0), \dot{\varepsilon}(0)) \in \mathcal{L}_{a}^{*}$, then $(\varepsilon(t), \dot{\varepsilon}(t)) \in \mathcal{L}_{a}^{*}, \forall t \in \mathbb{R}_{>0}$ and $\boldsymbol{\varepsilon}$ converges to $\mathbf{0}_{3 \times 1}$ exponentially. From the definition of $\boldsymbol{\varepsilon}$ and considering $(54)$, this is equivalent to say that $\left(\mathbf{D}^{\mathrm{B} / \mathrm{G}} ; \boldsymbol{\Omega}_{\mathrm{B}}^{\mathrm{B} / \mathrm{G}}\right)$ converges to $\left(\overline{\mathbf{D}}^{\mathrm{B} / \mathrm{G}} ; \mathbf{0}_{3 \times 1}\right)$ exponentially, thus completing the proof. 


\section{Nominal Simulation}

As a first step to evaluate the proposed method, this section presents a numerical simulation of the the proposed flight control system in nominal conditions.

It is worth noting at this point that both the aerostatic lift $\vec{F}^{b}$ and the restoring torque $\vec{T}^{b}$ depend on the value of $\rho_{\text {air }}$ and $\rho_{\text {helium. }}$. These densities in turn depend on the local temperature and pressure, as expressed by the Ideal Gas Law:

$$
\rho_{\mathrm{q}}=\frac{p}{R_{\mathrm{q}} T}
$$

where "q" can be replaced by either "air" or "helium", $p$ is the local pressure in $\mathrm{Pa}, T$ is the local temperature in $\mathrm{K}$, and $R_{q}$ is the specific gas constant $\left(R_{\text {air }}=286.9 \mathrm{~J} / \mathrm{Kg} / \mathrm{K}\right.$ and $R_{\text {helium }}=2077$ $\mathrm{J} / \mathrm{Kg} / \mathrm{K})$. In particular, in this section, the surrounding air and contained gas are suposed to have the same temperature and pressure; their values are $20^{\circ} \mathrm{C}$ and $101325 \mathrm{~Pa}$, respectively. Moreover, the air is assumed to be dry. Under such conditions, the air and helium densities are $\rho_{\text {air }} \approx 1.204$ $\mathrm{kg} / \mathrm{m}^{3}$ and $\rho_{\text {helium }} \approx 0.1664 \mathrm{~kg} / \mathrm{m}^{3}$. These values are used here to simulate the ground truth as well as to compute the control laws.

Subsection 4.1 describes the simulation and shows the adopted nominal parameters, while Subsection 4.2 presents and analyzes the simulation results.

\subsection{Plant and Controller Parameters}

For simulating the overall closed-loop flight control system represented in Figure 4, we use the models formulated in Section 2 as well as the control laws and control allocation proposed in Section 3. The simulator is implemented in MATLAB, using the forth order Runge-Kutta solver, with an integration step of $0.001 \mathrm{~s}$. Table 1 shows the nominal values of the hexa-rotor airship parameters. On the other hand, Table 2 presents the adopted controller parameters. The controller gains are tuned by trial and error, taking into account their proportional or derivative effect and considering the time-scale separation assumption as well.

In this paper, for obtaining simulation data that are consistent with a typical operation of MAVs, the proposed flight control system is commanded to follow a waypoint-based position 
Table 1: Physical parameters of the hexa-rotor airship.

\begin{tabular}{lll}
\hline \hline Description & Symbol & Value \\
\hline Force coefficient & $k_{f}$ & $1.2838 \times 10^{-5} \mathrm{~N} /(\mathrm{rad} / \mathrm{s})^{2}$ \\
Torque coefficient & $k_{\tau}$ & $3.0811 \times 10^{-7} \mathrm{Nm} /(\mathrm{rad} / \mathrm{s})^{2}$ \\
Maximum rotor speed & $\bar{\omega}_{\max }$ & $906.66 \mathrm{rad} / \mathrm{s}$ \\
Motor speed coefficient & $k_{\omega}$ & 1 \\
Motor time constant & $\tau_{\omega}$ & $0.01 \mathrm{~s}$ \\
Arm length & $l$ & $1 \mathrm{~m}$ \\
Volume of the balloon & $V$ & $5.3 \mathrm{~m}^{3}$ \\
CB-CM displacement & $d$ & $0.85 \mathrm{~m}$ \\
Semi-major axis & $\mathrm{a}$ & $1.25 \mathrm{~m}$ \\
Semi-minor axis & $\mathrm{b}$ & $0.8 \mathrm{~m}$ \\
Total inertia matrix & $\mathbf{J}$ & $\operatorname{diag}(2.0633,2.0651,1.9556) \mathrm{kgm}^{2}$ \\
Moment of inertia of the rotors & $J^{r}$ & $0.001 \mathrm{kgm}$ \\
Total empty mass & $m^{t}$ & $9.392 \mathrm{Kg}$ \\
\hline \hline
\end{tabular}

Table 2: Parameters of the attitude and position control laws.

\begin{tabular}{lll}
\hline \hline Description & Symbol & Value \\
\hline Proportional gain of the position controller & $\mathbf{K}_{1}$ & $\operatorname{diag}(0.5,0.2,0.7)$ \\
Derivative gain of the position controller & $\mathbf{K}_{2}$ & $\operatorname{diag}(2,1,3)$ \\
Proportional gain of the attitude controller & $\mathbf{K}_{3}$ & $\operatorname{diag}(20,50,1)$ \\
Derivative gain of the attitude controller & $\mathbf{K}_{4}$ & $\operatorname{diag}(10,20,1)$ \\
Maximum torque command & $\mathbf{T}^{\max }$ & {$\left[\begin{array}{llll}16.3 & 14.1 & 0.58\end{array}\right]^{\mathrm{T}} \mathrm{Nm}$} \\
Minimum force command & $\mathbf{F}^{\min }$ & {$\left[\begin{array}{llll}-5.8 & -5.8 & 2.7\end{array}\right]^{\mathrm{T}} \mathrm{N}$} \\
Maximum force command & $\mathbf{F}^{\max }$ & {$\left[\begin{array}{llll}5.8 & 5.8 & 54.6\end{array}\right]^{\mathrm{T}} \mathrm{N}$} \\
Maximum inclination angle & $\varphi^{\max }$ & 12 degrees \\
\hline \hline
\end{tabular}

trajectory. In this trajectory, the waypoints are connected by straight lines with length of $5 \mathrm{~m}$ and a constant desired velocity of $\bar{v}=0.5 \mathrm{~m} / \mathrm{s}$. Moreover, the heading angle command $\bar{\psi}$ is set to zero.

\subsection{Nominal Simulation Results}

Figures 6-8 are the results of the nominal simulation using the parameters of Tables 1-2. Figure 6 shows the effective position and the corresponding position command. In the ramp parts of the component trajectories, one can verify a steady-state error of about $2 \mathrm{~m}$. After finishing the ramps, there are no overshoots and the accommodation times (within $5 \mathrm{~cm}$ of the final value) are between $10 \mathrm{~s}$ and $14 \mathrm{~s}$. In the flat parts of the trajectory, the steady-state errors are zero. The large errors during the ramps and the long accommodation times reflect the vehicle's inherent low acceleration 
capability, which can be attributed to both the restricted inclination bound $\varphi^{\max }$ and the large inertia parameters.
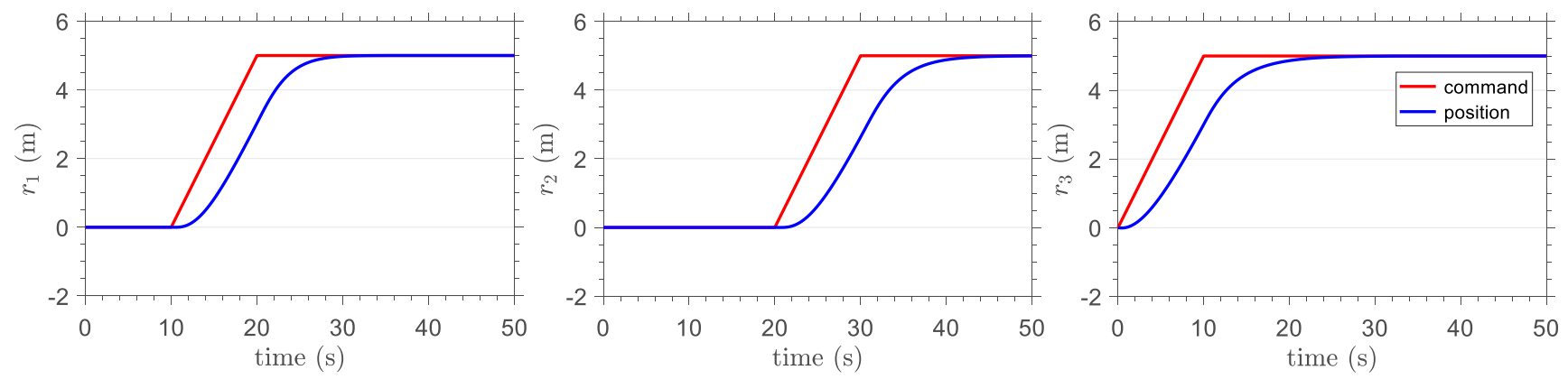

Figure 6: Nominal performance of the position control. It shows the controlled position components together with the respective command.

Figure 7 shows the effective and commanded attitude along the time using Euler angles in the 1-2-3 sequence. The attitude pitch and roll commands deviate from zero at the waypoints and the corresponding effective angles follow them with an apparently fast but attenuated response. In the yaw angle, one can see a very small and short transient starting at $20 \mathrm{~s}$; it is due to the gyroscopic coupling of the rotational dynamics, which becomes apparent when there exist a simultaneous motion in roll and pitch.
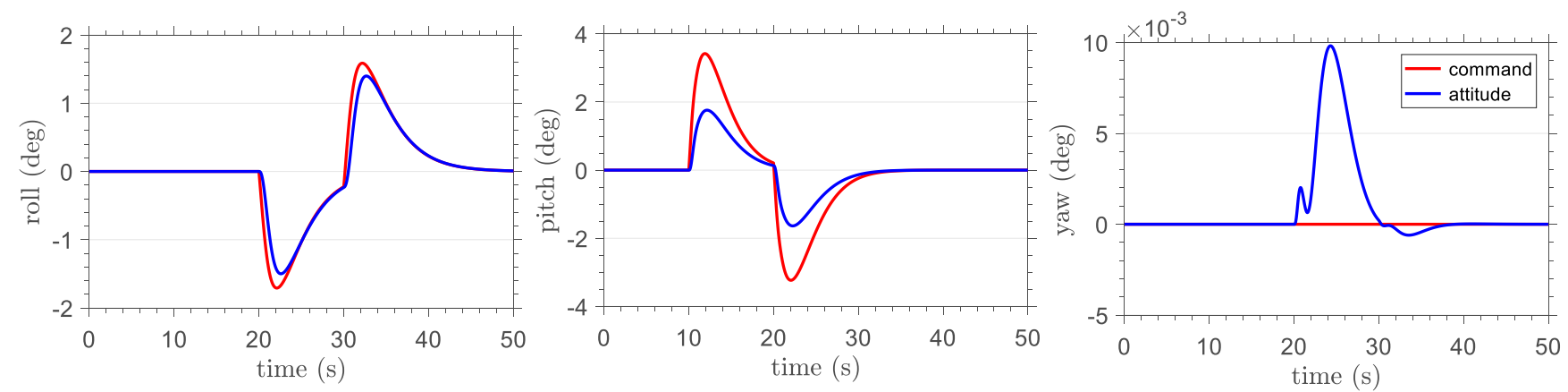

Figure 7: Nominal performance of the attitude control. It shows the controlled attitude variables together with the respective command.

Finally, Figure 8 presents the force magnitude command $\bar{F}^{c}$ computed by the position controller and Figure 9 shows the components of the torque command $\overline{\mathbf{T}}_{\mathrm{B}}^{c}$ generated by the attitude controller. 
There are transients at the instants the vehicle is commanded to ascend or to stop ascending, after which, $\bar{F}^{c}$ converges and stays around $42.1 \mathrm{~N}$. This force value is equivalent to the total weight of the vehicle, payload, and helium, minus the aerostatic lift. The force command $\bar{F}^{c}$ keeps far from its maximum bound, even during the ascending acceleration. On the other hand, the components of the torque command $\overline{\mathbf{T}}_{\mathrm{B}}^{c}$ deviate from zero at the waypoints, but stay inside their bounds $\pm \mathbf{T}^{\text {max }}$ with a large margin.

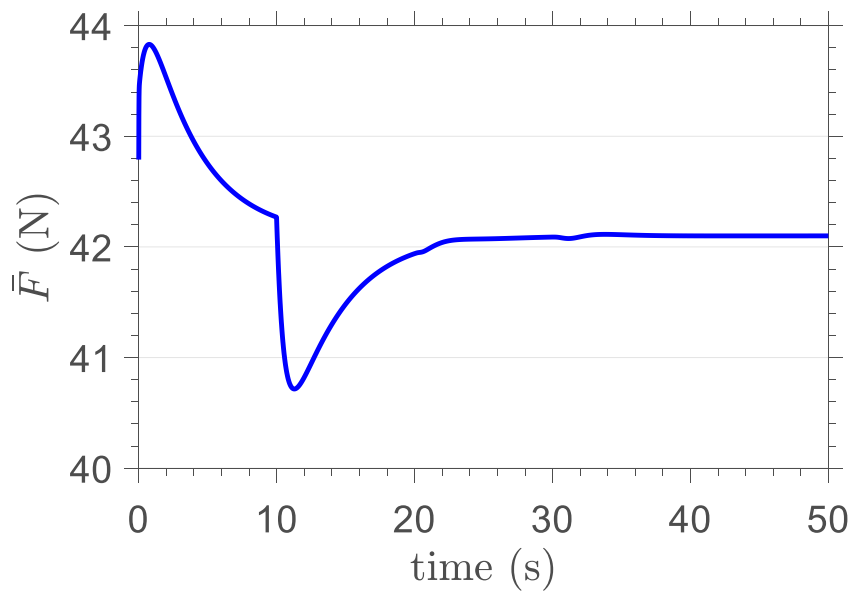

Figure 8: Force magnitude command provided by the position controller in the nominal simulation.

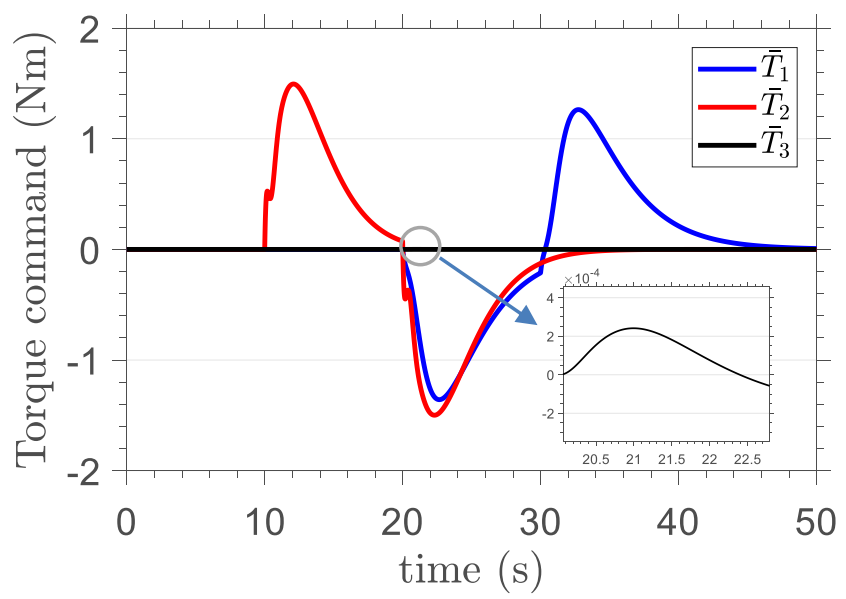

Figure 9: Torque command provided by the attitude controller in the nominal simulation. 


\section{Uncertainty Quantification}

The present section conducts an uncertainty quantification analysis of the flight control system under consideration using a parametric probabilistic approach [24, 25]. In this analysis, ambient the temperature and pressure are modeled as independent random variables and, based on such probabilistic models, for each flight realization, one new outcome is generated for both variables. For simulating the plant, the surrounding air and contained helium are assumed to have the same (varying) temperature and pressure. On the other hand, in the implementation of the control laws, their densities are maintained constant (and equal the respective nominal values; see Section 4).

\subsection{Probabilistic Model}

Let $(\Theta, \mathbb{Z}, \mathbb{P})$ be a probability space, where $\Theta$ is the sample space, $\mathbb{\Sigma}$ is a $\sigma$-algebra over $\Theta$, and $\mathbb{P}: \mathbb{Z} \rightarrow[0,1]$ a probability measure. In this stochastic framework, the local temperature $T^{l}$ and pressure $P^{l}$ are modeled by (independent) random variables $\mathbb{X}: \mathbb{Z} \rightarrow \mathbb{R}$ and $\mathbb{Y}: \mathbb{Z} \rightarrow \mathbb{R}$, respectively. We assume that the joint probability distribution of these random variables, $P_{\text {ZY }}(d x d y)$, admit a probability density function $(\mathrm{PDF})(x, y) \mapsto p_{\mathbb{K Y}}(x, y)$, with respect to $d x d y$. Similarly, the marginal distributions $P_{\nwarrow}(d x)$ and $P_{\curlyvee}(d y)$ are assumed as having marginal PDFs $x \mapsto p_{\nwarrow}(x)$, with respect to $d x$, and $y \mapsto p_{\curlyvee}(y)$, with respect to $d y$.

The maximum entropy principle [26] is adopted here as a way to specify the aforementioned distributions. This approach provides the least biased distributions in a scenario with little (or no) experimental information on a particular random variable [24]. In the present investigation, the random local temperature $\mathcal{X}$ and local pressure $\mathbb{Y}$ are both completely unknown beforehand. However, one should better specify for them resonable operational ranges, from which it is possible to immediately define the underlying supports. In this way, we assume from now on that $\mathbb{X}$ and $\mathbb{Y}$ take values in known positive finite intervals $\left[x_{1}, x_{2}\right] \subset(0,+\infty)$ and $\left[y_{1}, y_{2}\right] \subset(0,+\infty)$, respectively. Therefore, the maximum entropy principle is formulated here as the maximization of the Shannon entropy

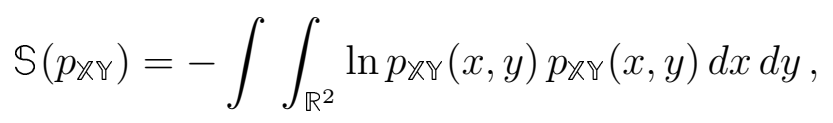


subject to the normalization constraint (which brings information about the supports)

$$
\int_{y_{1}}^{y_{2}} \int_{x_{1}}^{x_{2}} p_{\mathbb{K Y}}(x, y) d x d y=1
$$

By using the Lagrange multiplier method, one can solve the above optimization problem to obtain the following PDF

$$
p_{\text {XY }}(x, y)=p_{\mathbb{X}}(x) \times p_{\Im}(y),
$$

with

$$
p_{\text {\欠 }}(x)=\mathbb{1}_{\left[x_{1}, x_{2}\right]}(x) \frac{1}{x_{2}-x_{1}} \text { and } p_{\curlyvee}(y)=\mathbb{1}_{\left[y_{1}, y_{2}\right]}(y) \frac{1}{y_{2}-y_{1}} \text {, }
$$

where $\mathbb{1}_{X}(x)$ denotes the indicator function of the set $X$. Note that the above marginal PDFs correspond to uniform distributions on the intervals $\left[x_{1}, x_{2}\right]$ and $\left[y_{1}, y_{2}\right]$, respectively. Informally, the above result makes all sense, because since the minimum and maximum values are the only known informaion about the random parameters, the most reasonable choice for their distributions is the one that assigns equal weight to all their possible values.

Due to the randomness of $\mathbb{X}$ and $\mathbb{Y}$, the air density $\rho_{\text {air }}$ and the helium density $\rho_{\text {helium }}$ are also random variables [see equation (56)]. Further, since the aerostatic lift [see equation (7)] and the restoring torque [see equation (9)] depend on $\rho_{\text {air }}$ and $\rho_{\text {helium }}$, then the translational (14) and rotational (17) dynamic models are rather differential equations with random parameters and, consequently, their variables are stochastic processes. In particular, we choose here the minimum temperature $x_{1}=0^{\circ} \mathrm{C}$, the maximum temperature $x_{2}=40^{\circ} \mathrm{C}$, the minimum pressure $y_{1}=0.7739$ atm (at $2000 \mathrm{~m}$ and $0^{\circ} \mathrm{C}$ ), and the maximum pressure $y_{2}=1 \mathrm{~atm}$.

\subsection{Propagation of Uncertainties}

To compute the propagation of uncertainties of the random parameters $\mathbb{X}$ and $\mathbb{Y}$ through the control system dynamics, we employ the Monte Carlo (MC) method [27]. It consists in a stochastic solver that, based on $n_{s}$ realizations of the random parameters $\mathbb{X}$ and $\mathbb{Y}$, generates $n_{s}$ different realizations of the nominal closed-loop dynamics and computes the sample statistics of the respective

system responses. These statistics are then used to approximate the solution (or response) of the original stochastic system. 


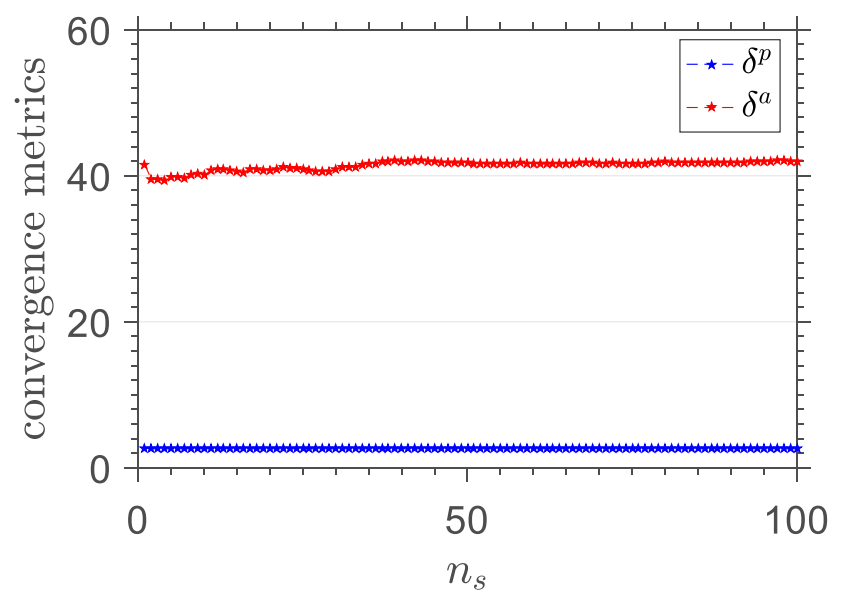

Figure 10: Convergence metrics for choosing the number of Monte Carlo realizations.

In order to guarantee the convergence of the MC solution to the true stochastic behavior of the system, a sufficient number $n_{s}$ of realizations must be considered. The reference [28] shows that such a convergence occurs in the mean-square sense if the following metrics converge in the point-wise sense:

$$
\begin{aligned}
\delta^{p} & =\left(\frac{1}{n_{s}} \sum_{n=1}^{n_{s}} \int_{t=0}^{t_{f}}\left(\left\|\mathbf{r}_{\mathrm{G}}^{\mathrm{B} / \mathrm{G}}(t, n)\right\|^{2}\right) d t\right)^{1 / 2}, \\
\delta^{a} & =\left(\frac{1}{n_{s}} \sum_{n=1}^{n_{s}} \int_{t=0}^{t_{f}}\left(\left\|\boldsymbol{\alpha}^{\mathrm{B} / \mathrm{G}}(t, n)\right\|^{2}\right) d t\right)^{1 / 2},
\end{aligned}
$$

where $n$ denotes an arbitrary MC realization, $t$ denotes the continuous time, $t_{f}$ is the final simulation instant, $\mathbf{r}_{\mathrm{G}}^{\mathrm{B} / \mathrm{G}}(t, n)$ is the $n$th realization of the vehicle's position at instant $t$, and $\boldsymbol{\alpha}^{\mathrm{B} / \mathrm{G}}(t, n)$ is the $n$th realization of the vehicle's attitude (in Euler angles 1-2-3) at instant $t$. Figure 10 shows a plot of the position (61) and attitude (62) convergence metrics versus $n_{s}$. One can see that after 50 realizations, both metrics show to converge. Therefore, in the problem under consideration, we consider $n_{s}=100$, which is sufficient for the convergence of the MC solution.

Figure 11 shows the position statistics (mean and confidence region). One can see that, as a consequence of the randomness in the temperature and pressure conditions, there is a very small variation in the $r_{1}$ and $r_{2}$ performance (less the $5 \mathrm{~cm}$ ), and a considerable variation in the $r_{3}$ performance (about $70 \mathrm{~cm}$ ). Figure 12 shows the statistics (mean and confidence region) of the attitude. The largest variations, which are very small (less than 0.3 degree), occur during the 
transients.
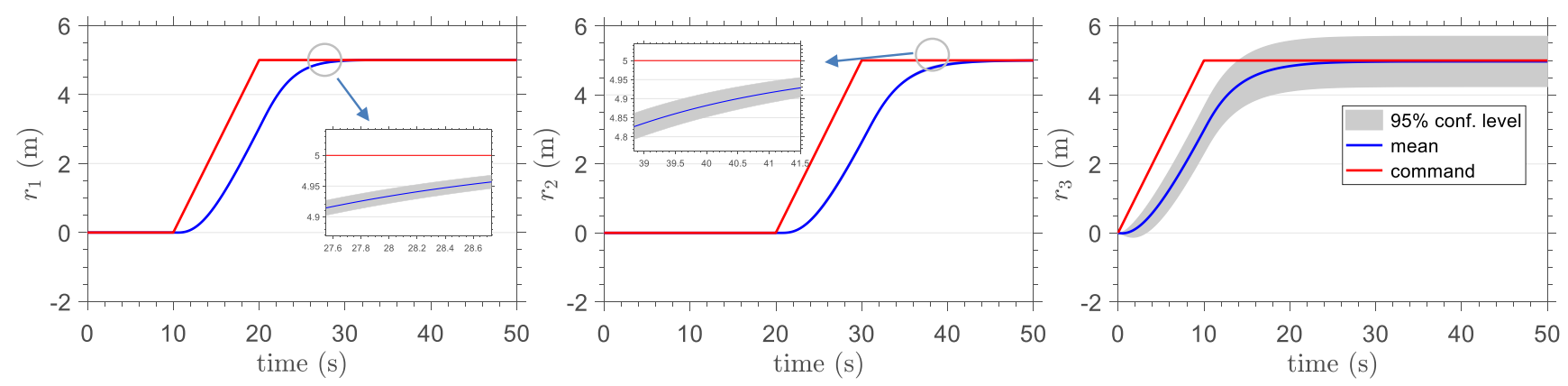

Figure 11: Position statistics (mean and confidence region) estimated by Monte Carlo method.
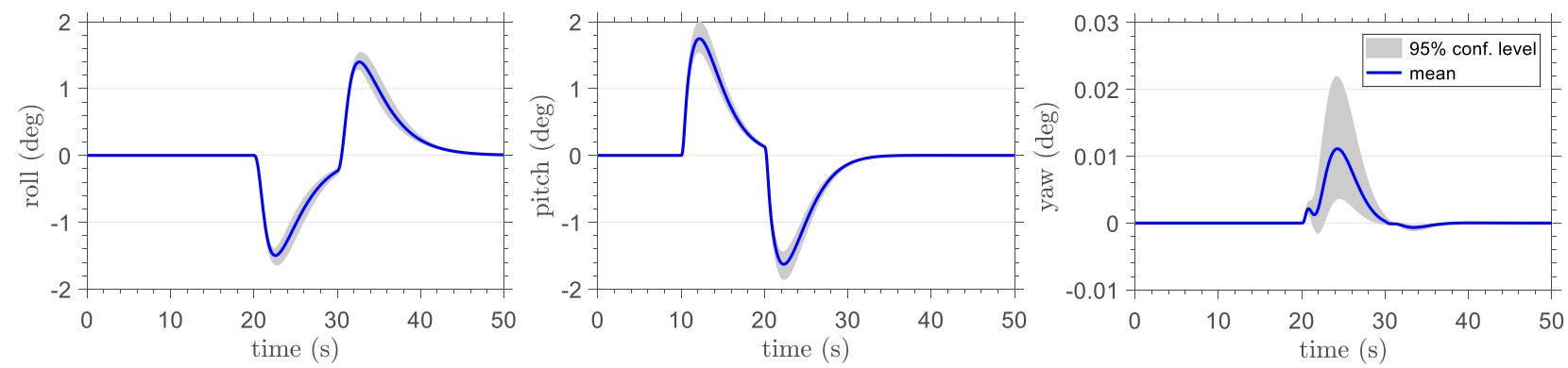

Figure 12: Attitude control error statistics (mean and confidence region) estimated by Monte Carlo method.

Figures 13 and 14 show the statistics (mean and confidence region) of the force magnitude command and torque command, respectively. One can observe a small random variation in the torque command components. On the other hand, there is a large variation in the force magnitude command; this is a direct consequence of the aerostatic lift variation (see Figure 15), which is proportional to the variation of the air density (see equation (7)). The extreme values of both commands keep inside their bounds with a good margin.

Figure 13: Torque command statistics (mean and confidence region) estimated by Monte Carlo method.

Figure 14: Force magnitude command statistics of the Monte Carlo simulation. 
Figure 15: Aerostatic lift statistics of the Monte Carlo simulation.

\section{Concluding Remarks}

The paper proposed a flight control system for a new micro aerial vehicle (MAV) resulting from the combination of a hexa-rotor airframe with a balloon filled with helium. This MAV is a straightforward attempt to extend the flight duration and load capacity of conventional multi-rotor vehicles by means of an aerostatic lift. On one hand, such a combination results in a very simple vehicle. On the other hand, even for indoor flight, its control system must be carefully designed to overcome the effects of a restoring torque (which does not occur in conventional multi-rotor vehicles), while being robust to variations in the local temperature and pressure operation conditions. The proposed flight control system is evaluated on the basis of both deterministic and stochastic simulations. In particular, the deterministic simulation shows that in nominal conditions, it is possible to control the vehicle's position to follow a desired waypoint-based trajectory with reference speed of $0.5 \mathrm{~m} / \mathrm{s}$. The obtained performance is sufficient accurate and fast for many MAV applications. On the other hand, in the stochastic simulation, the local temperature and pressure are modeled by uniformly-distributed random variables and the resulting performance is rigorously evaluated by Monte Carlo (MC) simulation. The variability verified in the position control performance can be considered small enough to dispense a special design of the control laws in order to suppress it. However, the variability shown in the attitude control error motivates a special robust control method for a better performance of the system in a stochastic sense.

\section{Acknowledgment}

The authors would like to thank all those people who indirectly contributed or motivated us to conduct the present investigation. In special, we are grateful to Mr. Luciano S. Araujo (from Elio Tecnologia), who designed and built the first prototype of the balloon-hexacopter and donated it to the Laboratorio de Robotica Aerea do ITA. The authors are thankful for the financial support provided by São Paulo Research Foundation (FAPESP) grant number XXX, Carlos Chagas Filho Research Foundation of Rio de Janeiro State (FAPERJ) under grants E-26/010.002.178/2015 and E-26/010.000.805/2018. 


\section{References}

[1] A. G. Khoury (Ed.), Airship Technology, Cambridge University Press, 2012.

[2] A. Elfes, S. S. Bueno, M. Mergerman, J. G. Ramos, A semi-autonomous robotic airship for environmental monitoring missions, Leuven, 1998, pp. 3449-3455.

[3] S. B. V. Gomes, J. G. Ramos, Airship dynamic modeling for autonomous operation, in: Proceedings of the 1998 IEEE International Conference on Robotics and Automation, Leuven, 1998, pp. 3462-3467.

[4] Y. Li, M. Nahon, I. Sharf, Airship dynamics modeling: A literature review, Progress inAerospaceSciences 47 (2011) 217-239.

[5] A. Moutinho, J. R. Azinheira, Stability and robustness analysis of the aurora airship control system using dynamic inversion, in: Proceedings of the 2005 IEEE International Conference on Robotics and Automation, Barcelona, 2005, pp. 2265-2270.

[6] T. Liesk, M. Nahon, B. Boulet, Design and experimental validation of a nonlinear low-level controller for an unmanned fin-less airship, IEEE Transactions on Control Systems Technology 21 (1) (2013) 147-161.

[7] E. Earon, C. A. Rabbath, J. Apkarian, Design and control of a novel hybrid vehicle concept, in: AIAA Guidance, Navigation, and Control Conference and Exhibit, Hilton Head, South Carolina, 2007, pp. 1-8.

[8] M. T. Frye, S. M. Gammon, C. Qian, The 6-dof dynamic model and simulation of the triturbofan remote-controlled airship, in: Proceedings of the 2007 American Control Conference, New York, 2007.

[9] N. Azouz, S. Chaabani, J. Lerbet, A. Abichou, Computation of the added masses of an unconventional airship, Journal of Applied Mathematics 2012 (2012) 1-19.

[10] E. Lanteigne, A. Alsayed, D. Robillard, S. G. Recoskie, Modeling and control of an unmanned airship with sliding ballast, Journal of Intelligent \& Robotic Systems 88 (2017) 285-297. 
[11] L. S. Araujo, Unmanned ellipsoid multi-rotor airship and respective method of construction. us patent, number 2018/0319476 a1. (2018).

[12] S. Bertrand, N. Guénard, T. Hamel, H. Piet-Lahanier, L. Eck, A hierarchical controller for miniature VTOL UAVs: Design and stability analysis using singular perturbation theory, Control Engineering Practice 19 (10) (2011) 1099-1108. doi:10.1016/j.conengprac.2011.05.008.

[13] T. Lee, M. Leok, N. H. McClamroch, Nonlinear robust tracking control of a quadrotor UAV on SE(3), Asian Journal of Control 15 (2) (2013) 391-408. doi:10.1002/asjc.567.

[14] X.-N. Shi, Y.-A. Zhang, D. Zhou, Almost-global finite-time trajectory tracking control for quadrotors in the exponential coordinates, IEEE Transactions on Aerospace and Electronic Systems PP (99). doi:10.1109/TAES.2017.2649258.

[15] D. A. Santos, A. Cunha Jr, Dynamic modeling and flight control of a balloon-quadcopter unmanned aerial vehicle, in: Proceedings of the XXXVIII Iberian Latin-American Congress on Computational Methods in Engineering, Florianopolis, 2017.

[16] M. Tuveri, A. Ceruti, P. Marzocca, Added masses computation for unconventional airships and aerostats through geometric shape evaluation and meshing, International Journal of Aeronautical and Space Sciences 15 (3) (2014) 241-257.

[17] R. Mahony, V. Kumar, P. Corke, Multirotor Aerial Vehicles: Modeling, Estimation, and Control of Quadrotor, IEEE Robotics \& Automation Magazine 19 (3) (2012) 20-32. doi:10.1109/MRA.2012.2206474.

[18] T. I. Fossen, Guidance and Control of Ocean Vehicles, Wiley, New York, 1998.

[19] P. G. Thomasson, Equations of motion of a vehicle in a moving fluid, Journal of Aircraft $37(4)$.

[20] Y. Li, M. Nahon, Modeling and simulation of airship dynamics, Journal of Guidance, Control, and Dynamics 30 (6) (2007) 1691-1700.

[21] I. B. Tuckerman, Inertia factors for ellipsoids for use in airship design (1925). 
[22] Y. Li, Dynamics modeling and simulation of flexible airships, phdthesis (2008).

[23] F. L. Markley, J. L. Crassidis, Fundamentals of Spacecraft Attitude Determination and Control, Springer, 2014.

[24] C. Soize, Stochastic modeling of uncertainties in computational structuraldynamics - recent theoretical advances, Journal of Sound and Vibration 332 (10) (2013) 2379 - 2395. doi:http://dx.doi.org/10.1016/j.jsv.2011.10.010.

[25] C. Soize, Uncertainty Quantification: An Accelerated Course with Advanced Applications in Computational Engineering, Springer, 2017.

[26] E. T. Jaynes, Information theory and statistical mechanics, Physical Review Series II 106 (1957) 620-630. doi:10.1103/PhysRev.106.620.

[27] R. Y. Rubinstein, D. P. Kroese, Simulation and the Monte Carlo Method, 3rd Edition, Wiley, 2016.

[28] C. Soize, A comprehensive overview of a non-parametric probabilistic approach of model uncertainties for predictive models in structural dynamics, Journal of Sound and Vibration 288 (2005) 623-652. doi:10.1016/j.jsv.2005.07.009. 